\title{
Measurement of Cross-Field Electron Current in a Hall Thruster Due to Rotating Spoke Instabilities
}

\author{
Michael S. McDonald*, Christopher K. Bellant ${ }^{\dagger}$, Brandon A. St. Pierre ${ }^{\ddagger}$ and Alec D. Gallimore ${ }^{\S}$ \\ Plasmadynamics and Electric Propulsion Laboratory (PEPL) \\ University of Michigan, Ann Arbor, MI USA 48109
}

\begin{abstract}
The first direct experimental measurements of electron current due to rotating spokes in a modern highpower Hall thruster are presented. A segmented anode consisting of 12 equally spaced azimuthal sections has been retrofitted onto the $\mathrm{H6}$ 6-kW class Hall thruster and operated at power levels up to 3 kilowatts. Independent discharge current measurements on each anode segment at 1 MHz and synchronous high-speed video of the discharge at $\mathbf{8 7 , 5 0 0}$ frames per second reveal that visible rotating spoke structures in the thruster channel correspond to local electron current oscillations with amplitude approximately $30 \%$ of the mean local discharge current through each segment. Discrete Fourier transforms of discharge current oscillations on each segment reveal peaks at spoke rotation frequencies an order of magnitude larger than at the well-known breathing mode frequency. The apparent dominance of the breathing mode in traditional Hall thruster discharge current frequency spectra is revealed to be an artifact of the use of a contiguous ring-shaped anode. Based on the magnitude of local discharge current oscillations on each segment, the magnitude of plasma density oscillations are inferred to be of the order of the mean plasma density and the net discharge current carried by the spoke mechanism is calculated to be up to $50 \%$ of the total thruster discharge current.
\end{abstract}

\section{Nomenclature}

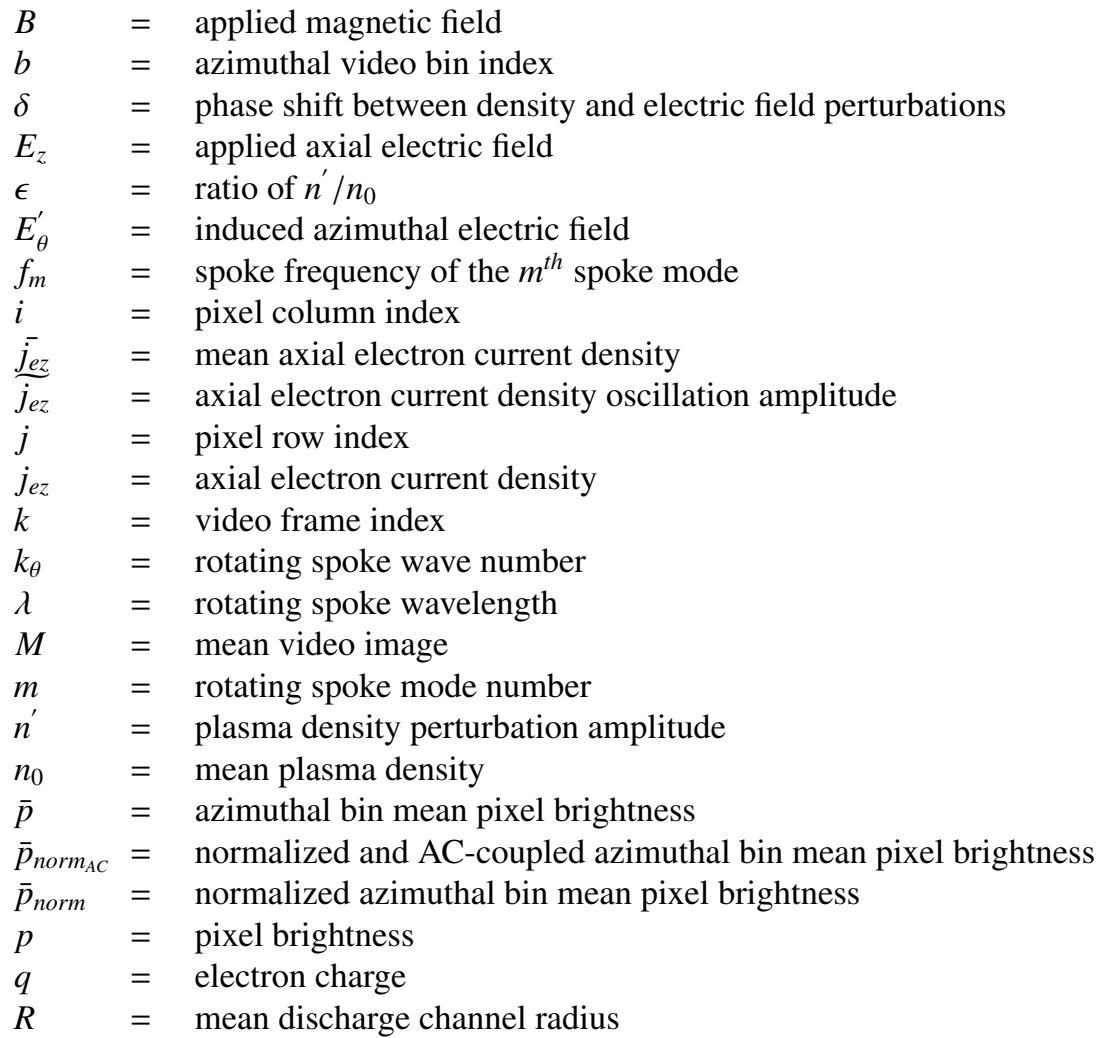

*Doctoral Candidate, University of Michigan, Applied Physics Program. msmcdon@ umich.edu. Student Member AIAA.

${ }^{\dagger}$ Undergraduate, University of Michigan Department of Aerospace Engineering.

¥Undergraduate, University of Michigan Department of Aerospace Engineering.

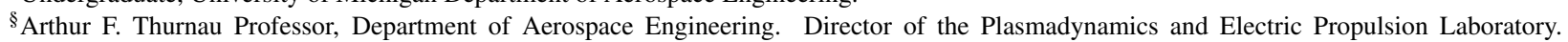
alec.gallimore@umich.edu. Fellow AIAA.

1

American Institute of Aeronautics and Astronautics 


$\begin{array}{ll}r & =\text { radial thruster coordinate } \\ \theta & =\text { azimuthal coordinate in Hall thruster channel } \\ \theta_{b} & =\text { azimuthal bin for video processing } \\ v_{e z} & =\text { axial electron velocity } \\ v_{m} & =\text { linear velocity of the } m^{t h} \text { spoke mode } \\ X_{k} & =\text { normalizing factor for } k^{t h} \text { video frame } \\ X_{t} & =\text { normalizing factor for discharge current at time } t \\ z & =\text { axial thruster coordinate }\end{array}$

\section{Introduction}

\section{A. Rising Hall Thruster Power Levels and the Need for an Electron Transport Model}

In February 1972 a Fakel SPT-60 with a power level of 500 watts (W) was the first Hall thruster to operate in space aboard the Soviet Meteor spacecraft. ${ }^{1,2}$ By the 1990s, thanks to sustained development in the USSR, the Fakel SPT-100 demonstrated operation on orbit at $1.35 \mathrm{~kW}$. At that time any Hall thruster with a discharge power level of a kilowatt or more could credibly have been considered a high-power Hall thruster. In August 2010 the BPT-4000 entered orbit with a power level of $4.5 \mathrm{~kW}$, and today it is unlikely that any thruster under the $5-10 \mathrm{~kW}$ range deserves that label. It is likely that in the next 5-10 years the high-power threshold will raise by another order of magnitude yet, as thrusters near to and in excess of $100 \mathrm{~kW}$ enter routine laboratory testing. Hall thrusters typically inhabit a discharge voltage envelope of a few hundred volts to achieve a specific impulses $\left(I_{s p}\right)$ in the 1000-3000 second range, so most of that power increase will go toward increased discharge current to raise propellant flow rates and thus thrust levels. With this coming increase in mass flow rate will come a requirement for increased vacuum test facility pumping speeds, but vacuum chambers capable of maintaining $10^{-5}$ Torr in the face of flowrates on the order of $100 \mathrm{mg} / \mathrm{sec}$ are exceedingly rare.

The need is upon us, in short, for better predictive Hall thruster models to enable thruster design with far higher fidelity before ground testing begins. The primary obstacle to predictive Hall thruster modeling remains the electron transport problem. While the theory of classical electron scattering and diffusion from collisions with heavy particles is well understood (see, for example, $\mathrm{Chen}^{3}$ ) classical theory has been known since the early development of Hall thrusters in the $1960 \mathrm{~s}^{4}$ to underpredict electron transport across the strong magnetic fields in Hall thrusters by orders of magnitude.

It is no exaggeration to say that electron dynamics in the Hall thruster plume affect nearly every aspect of thruster operation: the ease of electron transport across magnetic field lines sets equipotentials that guide ion acceleration and focusing, determining beam divergence and affecting channel erosion patterns; the extent to which they heat or cool during transport affects electron energies, altering ionization and excitation cross-sections and influencing propellant utilization efficiencies; and the ease with which the cathode electrons couple into the plume and set the cathode potential relative to the far-field plasma potential (the so-called cathode coupling voltage) determines the maximum attainable ion acceleration voltage, driving the largest part of losses in the voltage utilization efficiency. So long as Hall thruster models are governed by ad hoc transport coefficients derived more from empirical data matching than $a$ priori physical principles, there can be no truly predictive Hall thruster model. At present, in spite of an abundance of proposed mechanisms, accurate assessments of the magnitude of electron current due to any one mechanism are scarce, and comparative studies of their relative influence on a single thruster platform simply do not exist. Lacking a clear idea of what mechanism(s) are primarily responsible for transport, it is understandably difficult for the electric propulsion scientist to focus his or her theoretical and computational tools on the right targets.

In this paper we will quantitatively assess the fraction of discharge current in the near-anode region of a high-power annular Hall thruster attributable to a particular mechanism known as a rotating spoke instability. In the remainder of this section we explain the operating principle of the instability and our motivation for its detailed study. In Section II we outline a simple model for cross-field transport by the spoke mechanism, and in Section III describe an experiment involving an azimuthally segmented Hall thruster anode built to resolve rotating points of electron current deposition associated with visible spoke modes seen on high-speed camera. Section IV explains the use and analysis of high-speed imaging as a nonintrusive thruster diagnostic tool to capture the rotating spoke oscillations. In Section V we discuss the relevant experimental results including power spectra of the current oscillations, the link between visible rotating spokes on the high speed camera and current deposited to the anode segments, and a discussion of how operating the 
thruster with a segmented anode differs from a conventional, contiguous ring-shaped anode. Finally, in Section VI we calculate the amplitude of the cross-field electron current due to the rotating spokes based on the current oscillations measured on the segmented anode.

\section{B. Motivation for a Segmented Anode}

The recent advent of high-speed video cameras capable of framerates of tens to hundreds of thousands of frames per second (fps) has opened a new diagnostic window into Hall thruster plume behavior on plasma timescales. ${ }^{5-8}$ In particular, high-speed imaging of the Hall thruster discharge channel reveals rotating spokes in the plasma that manifest on camera as regions of elevated visible light emission rotating in the $E \times B$ direction at several hundred to a few thousand meters per second, with long wavelengths on the order of several to over ten centimeters. This instability appears omnipresent in Hall thrusters. It has been observed at the University of Michigan Plasmadynamics and Electric Propulsion Laboratory in several thrusters ranging over an order of magnitude in discharge power including the Busek BHT- $600^{9}$, Helicon Hall Thruster ${ }^{10}$, NASA M173v1 ${ }^{11}$, X2 nested channel Hall thruster ${ }^{12}$, and in the H6 6-kW Hall thruster that is the focus of this paper. Postprocessed video frames of a typical spoke instability in the H6 are shown in Figure 1.

We hypothesize that these visible spokes are the optical wake of excited ions and neutrals behind a passing electron density wave, and that plasma potential fluctuations in this wave create azimuthal electric field components capable of driving an axial $E \times B$ drift current, as in Figure 2. Just as the applied axial electric and radial magnetic fields $E_{z}$ and $B_{r}$ induce an azimuthal $E \times B$ electron drift (the Hall current that lends the thruster its name), an induced azimuthal electric field $E_{\theta}$ as in Figure 2 driven by a plasma instability would couple with the applied field $B_{r}$ to drive an axial drift component. Since these oscillations are periodic in the azimuth of the Hall thruster channel, traditional discharge current measurements on a conventional ring-shaped anode are unlikely to detect them, as the measurement is effectively integrated over the full $2 \pi$ of the Hall thruster channel. However, a segmented anode with isolated electrodes spaced azimuthally around the channel would be capable of resolving any azimuthally localized rotating points of electron current deposition. In this paper we measure the level of current attributable to low-frequency rotating spokes in the near-anode region by measuring the current to a segmented anode while synchronously acquiring high-speed video of the Hall thruster discharge. To this end we will postulate the existence of density and field fluctuations associated with the rotating spokes, following the findings of several previous authors. ${ }^{4,6,7,13}$
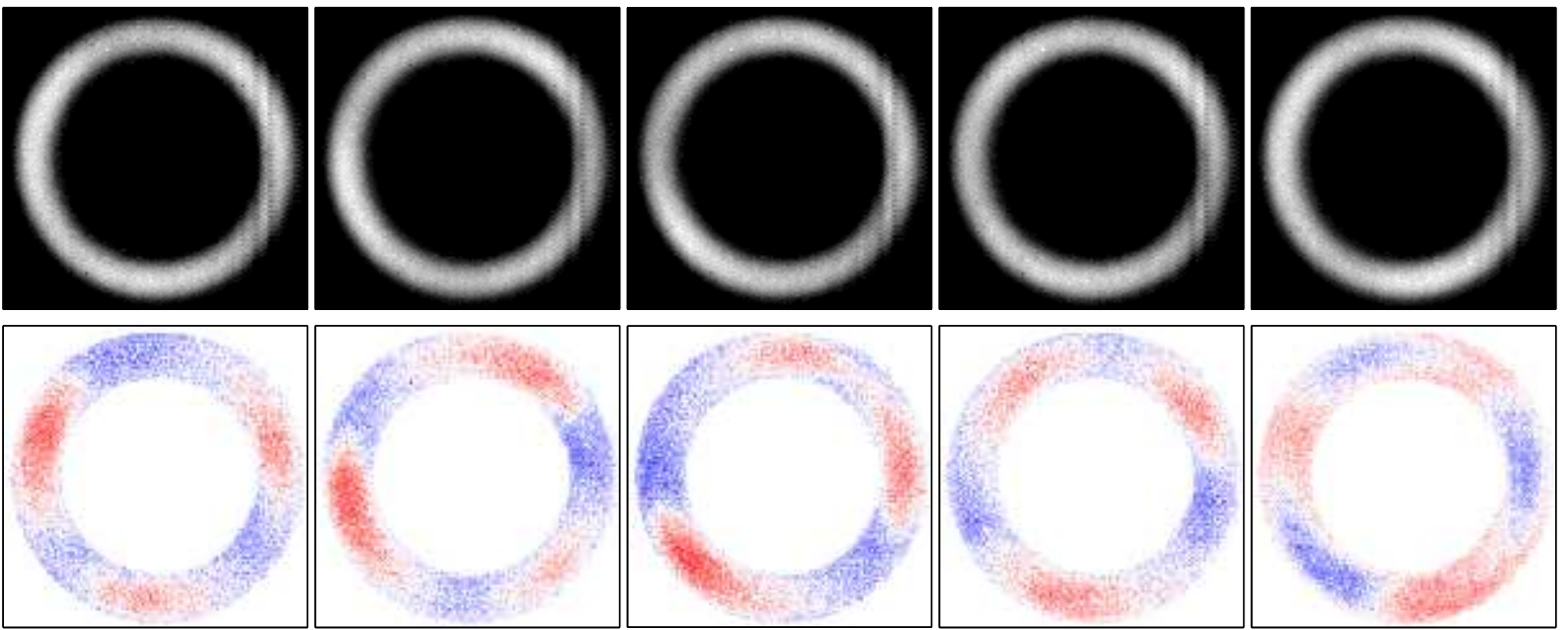

Figure 1. A series of high-speed video frames of a Hall thruster discharge (top) reveal rotating regions of elevated light emission after careful postprocessing (bottom), discussed in more detail in Section IV.B. In this image a coherent threefold rotating spoke structure propagates in the $E \times B$ or counterclockwise direction.

\section{Background on Rotating Spokes in Hall Thrusters}


Two types of mechanisms are generally proposed to explain anomalous (i.e., non-classical) cross-field electron transport in Hall thrusters: collisional transport via electron impact with thruster surfaces and turbulent transport via fluctuating azimuthal electric fields. Within the thruster channel the first mechanism is known as near-wall conductivity (NWC) and was first studied in the early Russian development of the Hall thruster. ${ }^{14-16}$ More recently direct electron trajectory modeling has suggested that surface collisions in the near-field with the thruster pole pieces also play a role. ${ }^{17}$ Various theories in support of the second mechanism have been proposed, including resistive instabilities due to electron collisions ${ }^{18}$, shear flow instabilities ${ }^{19}$, electrothermal instabilities $^{13,20}$, critical ionization velocity phenomena ${ }^{4,21}$ and many others, at frequencies ranging from the low kilohertz up into the megahertz. The proposed mechanisms number far greater than the quantitative assessments of transport due to any particular mechanism, and there is no clear picture of which mechanisms are dominant under which operating conditions.

Without a clear candidate mechanism, most Hall thruster models treat anomalous electron transport by artificially inflating the collision frequency with semi-empirical transport coefficients similar to Bohm diffusion. ${ }^{22}$ As Mikellides notes, "the variation of [these coefficients] from one thruster simulation to another is not based on first principles, which presents the biggest obstacle in advancing such models to fully predictive design tools for Hall thrusters." ${ }^{23}$

A rotating spoke instability was first shown to cause turbulent transport in Hall thrusters by Janes in $1966 .{ }^{4}$ In-situ Langmuir probes and floating emissive probes in his early laboratory model thruster demonstrated electron density and plasma potential fluctuations slowly rotating in the $E \times B$ direction with sufficient amplitude to account for the anomalous transport in the thruster. Janes also showed good agreement with the theory of Yoshikawa ${ }^{24}$. Rotating spokes have since been discussed sporadically in the literature, but only recently have any investigations been attempted that approach the remarkable thoroughess of this early work. With the cessation of Hall thruster funding in the United States by NASA in $1970^{25}$, there would be no further domestic studies of this mechanism for more than three decades.

In the focused Russian development of the Hall thruster during this period, Esipchuk investigated the rotating spoke mechanism in some detail. ${ }^{26} \mathrm{He}$ linked the mode to incomplete ionization and found that it abated or disappeared at higher power levels, appearing more commonly at low discharge voltages relative to the so-called saturation voltage, which Zhurin describes as the "knee" in a plot of discharge current vs. increasing discharge voltage at fixed magnetic field settings. ${ }^{1}$ Both of these tendencies observed by Esipchuk differ sharply from the behavior of the rotating spokes observed on the $\mathrm{H} 6$ via high-speed imaging. The spokes tend to become more stable and of larger visible amplitude at higher discharge voltages for a given discharge current, with the strongest spoke mode occurring at the $600 \mathrm{~V} 6-\mathrm{kW}$ operating condition, the highest voltage and power level imaged on the H6 to date. This condition operates at a total efficiency (i.e., including cathode flow) of 65\%. Moreover, for fixed magnetic field settings, the spoke modes in the H6 become more pronounced at higher voltage, also opposite the tendency noted by Esipchuk.

In the United Kingdom in the late 1970s Lomas linked electron current in a high-current-density hydrogen Hall accelerator intended for controlled fusion work to a rotating spoke or "streamer". ${ }^{13}$ Lomas detected the spoke via optical and electrostatic probes, measured an azimuthal electric field fluctuation in phase with a density fluctuation in the spoke, and calculated that $20-70 \%$ of the $100 \mathrm{~A}$ discharge was carried by this mechanism. Lomas also used the electrothermal theory of Nelson ${ }^{20}$, which had already been applied to streamer modes in MHD generators, to describe a dispersion relation for two modes, a low-frequency spoke mode and a high-frequency ( $5 \mathrm{MHz}$ ) streamer mode.

Examination of the spoke mode in the United States resumed in the early 2000s at Stanford University, where Chesta observed several spoke-type instabilities in low-voltage Hall thruster discharges of approximately 80-200 V using azimuthally spaced probes, though he did not conclusively link them to electron transport. ${ }^{27}$ Chesta's efforts are noteworthy as the first since the work of Lomas to attempt a model of the Hall thruster discharge channel in a 2-D axial-azimuthal ( $\mathrm{z}-\theta$ ) formulation, using numerical techniques to solve the complicated dispersion relations and carry out stability analyses on the resulting modes. ${ }^{28}$ Like the earlier work of Janes and Lomas, Chesta ultimately attributed the spoke formation to electrothermal processes such as ionization, though the detailed mechanisms of this

4

American Institute of Aeronautics and Astronautics 
formation were left and still remain unclear. Later work at Stanford by Meezan would experimentally characterize an anomalously high electron mobility near the thruster acceleration region and, by association with large plasma fluctuations also measured in that region, make a correlative argument that the plasma fluctuations were linked to the transport. ${ }^{29}$ However, this argument extended well beyond the frequency band of rotating spokes, encompassing all density fluctuations measured by Chesta via a Langmuir probe in ion saturation sampled at $800 \mathrm{kHz}$.

The most recent investigation of the spoke modes has been at Princeton University, where in a parallel and independent effort Parker used high-speed video in a manner similar to this work to detect a spoke instability in a small low-power cylindrical Hall thruster (CHT). Spokes were found to be only occasionally present in the CHT, and their appearance linked to a drastic decrease in thruster efficiency associated with large increases in backstreaming electron current. ${ }^{6}$ Further work by Ellison used in-situ electrostatic probes embedded in the CHT channel to measure density and field fluctuations consistent with axial transport, and again in a parallel but independent effort from our own, created a small 4-element segmented anode and estimated approximately half the discharge current passed through a rotating spoke. ${ }^{7}$

However, again the results of the H6 differ markedly from those observed in other devices - whereas the transition observed by Parker showed increased electron current and poorer performance due to the onset of rotating spokes, in the $\mathrm{H} 6$ to the extent that such transitions have been observed the onset of rotating spokes generally corresponds to improved performance. Ultimately, as Choueiri notes in his overview of Hall thruster oscillations, "the detailed physics of this [rotating spoke] mode in the Hall thruster plasma remain largely unexplored." 30

\section{Theory of Turbulent Cross-Field Transport}

The basic principles that will be used to analyze cross-field transport in this paper follow the prescriptions of Janes and Yoshikawa. Yoshikawa derived an explicit analytical description of cross-field electron transport for the case of an azimuthal perturbation in plasma density and concomitant azimuthal electric field perturbation, as described above.

The net transport across the magnetic field by this mechanism, if it exists, is a second-order effect in the perturbed quantities. Consider a simple azimuthal fluctuation $E_{\theta}$ with an associated density fluctuation imposed on top of the typical $E_{z}$ and $B_{r}$ Hall thruster fields of the form

$$
\begin{gathered}
E_{\theta}=E_{\theta 0}+E_{\theta}^{\prime} \sin (\theta)=E_{\theta}^{\prime} \sin (\theta) \\
n=n_{0}+n^{\prime} \sin (\theta)
\end{gathered}
$$

where $E_{\theta 0}$ is zero since Faraday's law requires $\oint E \cdot d \vec{l}$ disappear in the absence of time-varying magnetic fields. We neglect any magnetic fields induced by the Hall current or thruster transients, following simulations by Haas showing that these are negligible in comparison with the applied field. ${ }^{31}$ The axial electron current density $j_{e z}$ due to the new drift current in the $E_{\theta} \times B$ direction is given by

$$
j_{e z}(\theta)=n q v_{e z}=n q \frac{E_{\theta}}{B}=\frac{q}{B}\left(n_{0} E_{\theta}^{\prime} \sin (\theta)+n^{\prime} E_{\theta}^{\prime} \sin ^{2}(\theta)\right)
$$

where we have chosen to ignore any radial variation of the axial electron current density, i.e., $j_{e z}(r, \theta)=j_{e z}(\theta)$ and we consider the electron velocity to be purely in the direction of $\vec{E} \times \vec{B}$ with an axial component $E_{\theta} / B$ under an adiabatic assumption that on the timescales we are interested in, the electron cyclotron motion is unimportant. To find the average current density $\overline{j_{e z}}$ over the channel we integrate over $\theta$ from 0 to $2 \pi$. The $\sin (\theta)$ term on the RHS of Equation 1 disappears under this integration, leaving only the contribution due to the product of two primed quantities:

$$
\overline{j_{e z}}=\frac{1}{2 \pi} \int_{0}^{2 \pi} j_{e z}(\theta) d \theta=\frac{q}{2 B} n^{\prime} E_{\theta}^{\prime}
$$

Janes notes that, based on Yoshikawa's analytical expressions, it is possible to express the azimuthal field fluctuation amplitude as a function of the density fluctuation and the applied axial field:

$$
E_{\theta}^{\prime}=\frac{1}{4} \pi \frac{n^{\prime}}{n_{0}} E_{z}
$$


Substituting this formula into Equation 1 above,

$$
\begin{gathered}
j_{e z}(\theta)=\frac{q \pi}{4 B} E_{z}\left(n^{\prime} \sin (\theta)+\frac{\left(n^{\prime}\right)^{2}}{n_{0}} \sin ^{2}(\theta)\right) \\
\overline{j_{e z}}=\frac{q \pi}{8} \frac{E_{z}}{B} \frac{\left(n^{\prime}\right)^{2}}{n_{0}}
\end{gathered}
$$

In the event that the peaks in the electric field and density are not perfectly in phase, i.e., $E_{\theta}=E_{\theta}^{\prime} \sin (\theta)$ and $n=n_{0}+n^{\prime} \sin (\theta+\delta)$, the effect is only to introduce a $\cos (\delta)$ term into Eqn. 2. For approximately in phase signals the relation is still accurate to second order in $\delta$. In this experiment the azimuthally segmented anode isolates the amplitude of $j_{e z}(\theta)$ and enables calculation of $n^{\prime}$. The quantities $n_{0}, E_{z}$ and $B$ are already known in the H6 so with $n^{\prime}$ we can determine $\bar{j}_{z}$ and the fraction of the total discharge current due to this mechanism.

\section{Experimental Setup}

\section{A. Facility and Thruster}

All tests presented in this paper take place on the H6 Hall thruster in the Large Vacuum Test Facility (LVTF) at the University of Michigan Plasmadynamics and Electric Propulsion Laboratory (PEPL). The LVTF is a cylindrical stainless steel-clad chamber $9 \mathrm{~m}$ long and $6 \mathrm{~m}$ in diameter. It is maintained at high vacuum by seven TM-1200 cryopumps with a combined pumping speed of $210,000 \mathrm{~L} / \mathrm{s}$ on xenon.

The H6 Hall thruster is a nominal 6-kW class Hall thruster with a nominal operating condition at $300 \mathrm{~V}$ discharge voltage and $20 \mathrm{mg} / \mathrm{s}$ anode flow rate with a $7 \%$ cathode flow fraction. This thruster has been widely documented elsewhere, for example by Reid ${ }^{32,33}$, and is notable for its high anode efficiency. During operation with a contiguous anode at the $300 \mathrm{~V}, 10 \mathrm{mg} / \mathrm{s}$ operating condition presented here, the anode efficiency is $64 \%$ and the total efficiency including cathode flow is $60 \% .{ }^{34}$ Operating at $800 \mathrm{~V}$, the thruster has demonstrated total efficiency of over $70 \% .{ }^{35}$ Performance measurements have not been taken with the segmented anode, but comparison of the segmented anode discharge current and FASTCAM video with normal contiguous anode operation will be presented. For the $10 \mathrm{mg} / \mathrm{s}$ anode flow rate and $0.7 \mathrm{mg} / \mathrm{s}$ cathode flowrate used, the base pressure of the chamber corrected for xenon was $7.7 \times 10^{-6}$ Torr.

\section{B. Segmented Anode}

The segmented anode used in this experiment is a set of 12 independently monitored electrodes biased at anode potential and equally spaced azimuthally at the rear of the annular Hall thruster channel. The anode segments are machined from a single solid ring of 300-series stainless steel and are electrically isolated from one another within the thruster assembly by boron nitride (BN) spacers (see Figure 3).

\section{Segmented Anode Design}

The segmented anode was designed with the express goal of making minimal if any changes to the original H6 structure. The existing magnetic circuit and discharge channel were left completely intact, e.g., no holes were drilled through the pole pieces or channel walls. Installation is instead an entirely reversible procedure with the segmented anode installed over the original anode, which is retained as an electrically floating gas distributor. Current-carrying lines are routed out the rear of the thruster through clearance in existing holes for the anode mechanical support studs.

The stainless steel anode segments are held in place and mutually isolated from the original H6 anode and from each other by insulating boron nitride $(\mathrm{BN})$ spacers. The BN spacers are in turn held in place on the original contiguous H6 anode by means of a twist-and-lock mechanism similar to channel nuts in unistrut framing. The extra height of the spacers and segments over the original anode plus the space behind the anode required to route the current-carrying wires out the anode support holes effectively shortens the discharge channel to $90 \%$ of its original unmodified length as measured from the anode surface to the exit plane, and the BN spacers rise an additional 1.5 mm above each anode segment. The introduction of the segmented anode also effectively converts the H6 from an axial to a radial neutral injection scheme, rerouting the flow around the segments to the inner and outer channel radii. In the present work the 

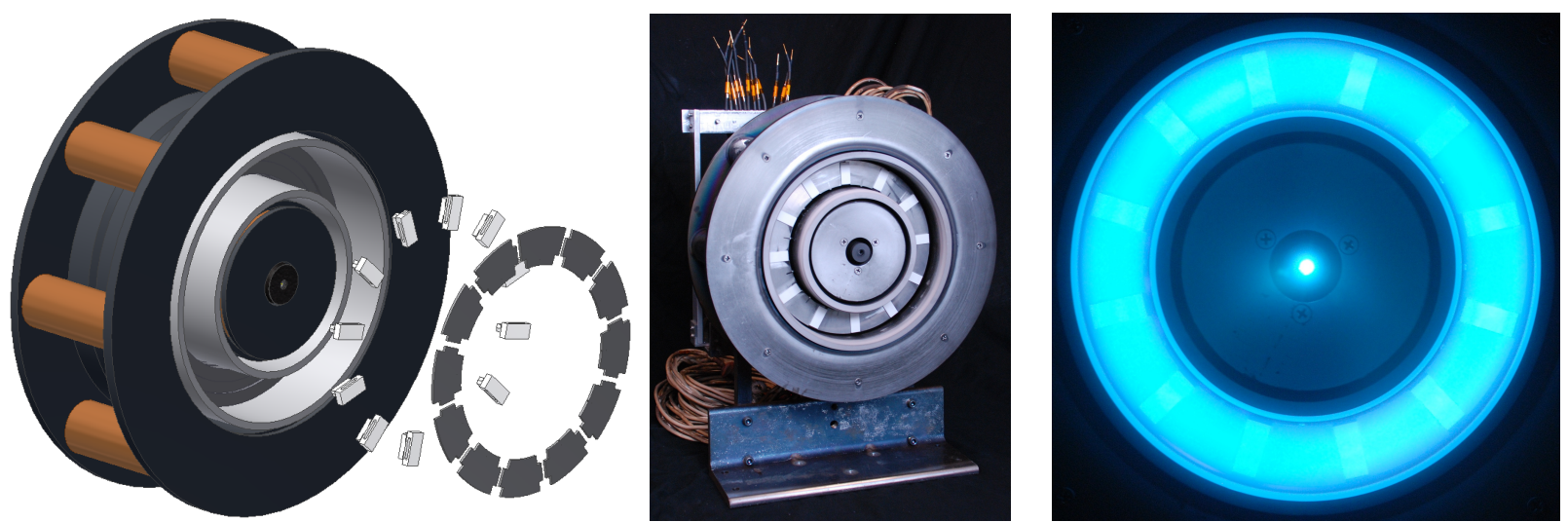

Figure 3. Left, an exploded view of the H6 Hall thruster with segmented anode, showing the 12 boron nitride spacers and 12 stainless steel anode segments. Middle, the thruster fully assembled with the segmented anode installed. The 12 current lines for the segments are visible rising out the back of the thruster. Right, an axial view of the thruster firing with the segmented anode installed. The white boron nitride spacers are more reflective than the stainless steel anode segments, and so appear brighter in the image. This vantage point is also used to record the high-speed video presented in Section V.B.

implications of this change in neutral flow dynamics are not explored, though a comparison between operation with and without the segmented anode is made in Section V.D.

Copper magnet wires $1.0 \mathrm{~mm}$ in diameter (.040" or $18 \mathrm{AWG)}$ are attached to each segment and mechanically held in place by a crimp connection to a $1 / 8$ " length $0-80$ thread stud that secures into a tapped hole on the inner diameter of each anode segment. As noted above these lines are routed out the rear of the thruster through clearance in the holes for the original anode's support studs. At that point they connect to 22 AWG coaxial cables that run 25 feet to the chamber feedthrough and another 3 feet outside the chamber to the measurement circuitry.

The tight fit between the current carrying wires and the inner radius of the discharge channel make this the region most susceptible to breakdown between the segments and the original anode. This is also the hottest region during thruster operation, as the wires experience both Ohmic heating from the discharge current through the wire and direct heating from segments as the discharge current falls through the anode sheath. Isolation from the floating anode is maintained by polyamide/polyimide lacquer on the magnet wire, fiberglass tape and a layer of mica insulation. The magnet wire alone is capable of isolation up to $2 \mathrm{kV}$ at atmosphere and room temperature, but is only rated to $200 \mathrm{C}$. The fiberglass tape holds the mica in place as an additional high-temperature insulator. Finally, the entire outer surface of the original anode is coated in a layer of polyimide (Kapton) tape to prevent glow discharges or Paschen breakdown from occurring in the narrow gaps and relatively high pressure of the rear of the discharge channel.

\section{Measurement Circuitry}

The measurement circuitry for the segmented anode is necessarily biased to anode voltage and in series with the main discharge. As a result all current sensors are contained within a Plexiglas box during the experiment. The current to each anode segment is monitored by a F.W. Bell NT-5 magneto-resistive current sensor. The NT-5 is a nominal 5 amp current sensor capable of handling current spikes up to $50 \mathrm{~A}$ and with voltage isolation to $3500 \mathrm{~V}$.

The specified NT-5 measurement bandwidth is DC to $100 \mathrm{kHz}$, and all sensors are sampled at $1 \mathrm{MHz}$. Due to the $100 \mathrm{kHz}$ bandwidth limit on the NT-5, all current measurements presented in this paper have been passed through an anti-aliasing low-pass filter with a cutoff frequency at $100 \mathrm{kHz}$.

Owing to the large number of current signals to be acquired, 8 segments are sampled on a General Standards PMC-16AISS8AO4 8-channel 16-bit data acquisition board with independent analog-to-digital converters on each channel, while the remaining 4 segments are captured to internal memory on an Agilent DSO-X 3024A 4-channel digital oscilloscope operating in an enhanced 9-bit acquisition mode.

In addition to individual segment current measurements through the NT-5 sensors, two split-core Hall probe measurements of the AC-coupled discharge current oscillations were also recorded. The first, with a Tektronix TCP303 sensor and TCPA300 amplifier, was just upstream of the measurement circuitry on the anode side, measuring the full oscillations of the discharge current from the supply before the lines split off to each segment. The second, with a Tektronix TCP312 sensor and TCPA300 amplifier, was on the cathode side of the circuit. These signals were recorded 
at $180 \mathrm{MHz}$ and provided high-bandwidth confirmation of the oscillations observed with the FW Bell NT-5's.

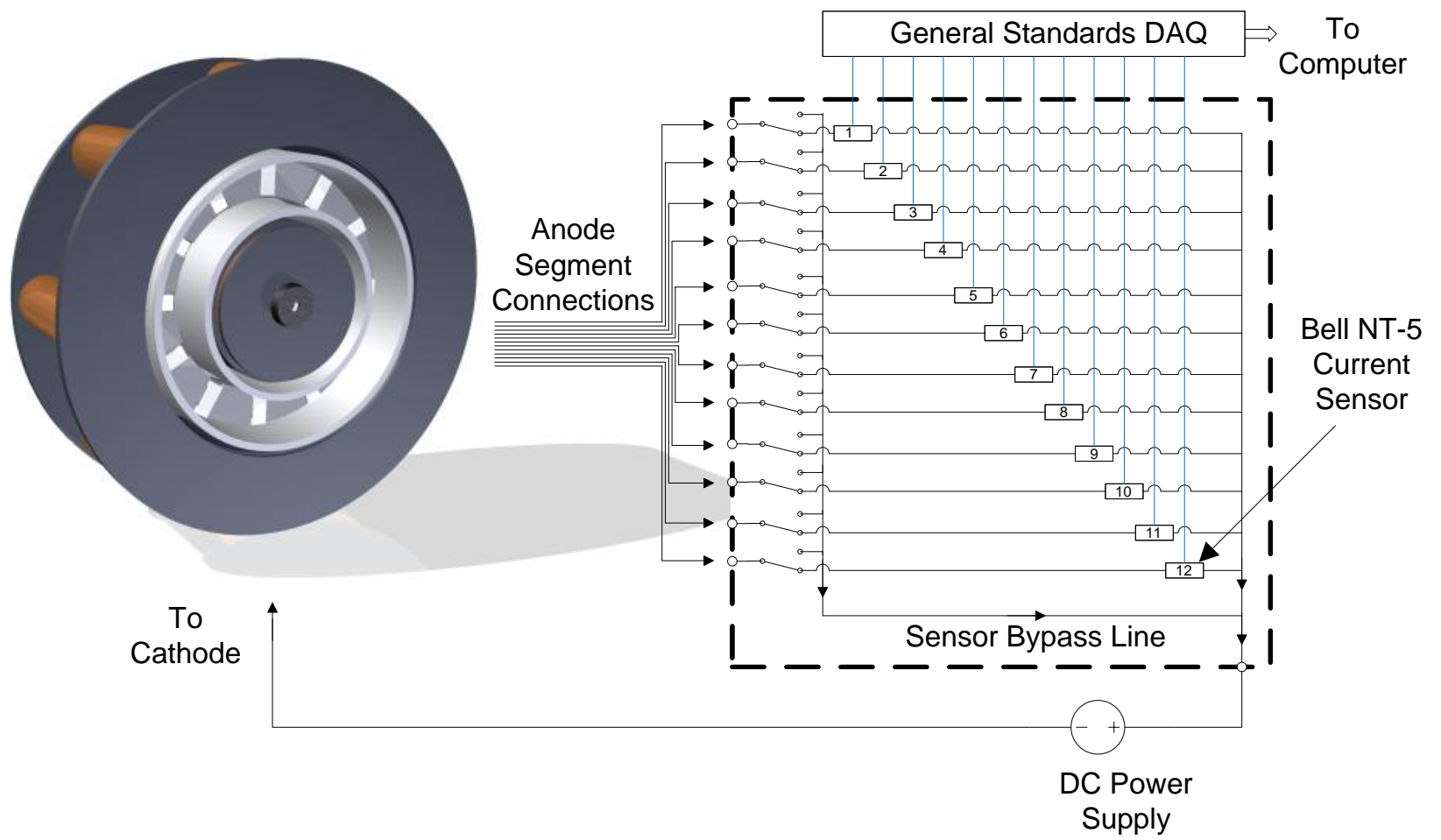

Figure 4. The segmented anode measurement circuit, contained in a Plexiglas box denoted by the dotted lines, consists of 12 separate current carrying lines to each anode segment. The lines pass through 12 FW Bell NT-5 magnetoresistive current sensors before rejoining into a single line to the main power supply. Each line additionally has a switch to bypass the sensors and the data acquisition system during thruster startup and shutdown transients.

\section{Segmented Anode Operation}

Early segmented anode tests were prone to overheating and breakdown. This tendency increased over time, as the thruster heated up and the insulation weakened. These breakdowns were visible as sporadic flashes on the high speed video as well as spikes in the discharge current signals. During the tests presented here the H6 was operated in a semi-pulsed fashion to preserve electrical isolation. The thruster was ignited in glow discharge mode and the magnetic field was slowly ramped up from zero to full strength (a so-called "soft start"). To prevent startup transients from causing arcing within the measurement circuitry, bypass switches (shown in Fig. 4) routed currents on startup around the sensors and directly from the power supply to the anode. Only after the thruster was lit and the magnetic field applied were the bypass switches disengaged and current allowed to flow through the Bell NT-5 current sensors.

This process took only a few minutes, and as soon as it was complete data acquisition from the current sensors and FASTCAM was initiated. Immediately after data acquisition the thruster was shut down. As a result, the discharge current is elevated during these tests because the discharge channel is still undergoing outgassing and bakeout, and thermal steady state has not yet been reached. While not ideal, the discharge is quiescent on the high speed video and on the segmented anode current measurements, bearing out that this technique was successful.

\section{High speed camera}

All images shown in this paper are taken with a Photron SA5 FASTCAM with a Nikon ED AF Nikkor 80-200mm lens operated at its maximum aperture of $\mathrm{f} / 2.8$. The SA5 is capable of full megapixel $1024 \times 1024$ resolution up to 7,000 frames per second (fps), with a peak framerate over 1,000,000 fps at lower resolutions. For this test it was operated at 87,500 fps at a $256 \times 256$ pixel resolution.

The FASTCAM views the thruster axially through a quartz viewport with an interior sacrificial glass plate cover from approximately 6.5 meters downstream. The thruster is laser-aligned with the camera in the vertical plane, and 
is slightly angled and offset from chamber centerline as a result in order to present as direct a view to the high-speed camera as possible. In the horizontal plane the viewport is raised above the thruster mounting surface and all high speed video and images are taken from approximately 2.5 degrees above the horizontal level of the thruster.

\section{High Speed Imaging as a Hall Thruster Diagnostic}

The high speed and high resolution of the FASTCAM conspire to generate very large file sizes. A typical video analyzed in this paper has 87,500 frames, of total duration 1 second, each with $256^{2}=65,536$ pixels. At the full 12-bit resolution of the camera, this corresponds to a file size of approximately $8 \mathrm{~GB}$. These large files lend themselves far better to statistical analysis than detailed examination of individual images, and for the most part in this work we will focus on power spectra derived from discrete Fourier transforms (DFTs) of the thruster discharge channel to characterize the modes and frequencies of oscillations. The DFT is calculated using the fast Fourier transform (FFT) algorithm and is also sometimes simply referred to as a FFT.

\section{A. Fourier analysis of thruster high speed video}

Consider a video as a 3-dimensional matrix of pixel values, with image pixel brightness is given as a function of position and time,

$$
p=p(i, j, k)
$$

where $i$ and $j$ denote the pixel column and row within a single frame and $k$ is an index denoting the frame itself within the video as a whole. In general, just as the DFT of a 1-D signal like the discharge current may be shown as as a 1-D line on a coordinate grid, the full DFT of a 3-D signal like a video requires a 3-D representation. This is neither practical nor terribly informative, so we will simplify this 3-D representation in several ways.

Since this work focuses on an azimuthally propagating instability, we will consider the videos in polar coordinates, i.e.,

$$
p=p(r, \theta, k)
$$

We will furthermore restrict our analysis to a specific region, the discharge channel, where we will choose to neglect the radial dependence of pixel brightness. To accomplish this we isolate the annular region of the discharge channel in each video frame and further divide the 360 degrees of azimuthal angle into several discrete angular bins, denoted by the index $b$ below, and deal with averaged pixel intensities $\bar{p}$ in each bin defined as

$$
\bar{p}\left(\theta_{b}, k\right)=\frac{1}{N_{b}} \sum_{r=R_{i}}^{R_{0}} p\left(r, \theta_{b}, k\right)
$$

where $N_{b}$ is the number of pixels in the $b^{\text {th }}$ bin. This reduces the video from a 3-D matrix of images to a 2-D plane in angle and time, dubbed the "spoke surface" (see Figure 5).

The spoke surface is amenable to a 2D DFT which can be powerfully interpreted in terms of the wave structures present in the thruster discharge. To compare, a 1D DFT breaks a 1D signal down into a finite series of sinusoidal basis functions with different amplitudes. These are necessarily functions of the single signal variable, usually time, and represent oscillations in time. By contrast, the 2D DFT breaks down a 2D signal, in the case of the spoke surface a function of time and angle, into three different kinds of oscillations. The first two are oscillations purely in time or purely angular, but these are limiting cases of the more general oscillations the 2D DFT reveals, which are oscillations linking both time and angle; i.e., waves propagating azimuthally around the discharge channel in time.

The DFT of the spoke surface generates a plane like the one at left in Figure 6. The DFT is of the same dimensions as the original discrete signal, so for a 1-second signal of 87,500 images, there are rows in the DFT corresponding to frequencies from $1 \mathrm{~Hz}$ up to $87,499 \mathrm{~Hz}$, with one row reserved for DC or $0 \mathrm{~Hz}$ signals. Likewise, for the 180 azimuthal bins used in this paper, there are columns corresponding to sinusoids with wavelengths from 0 to $179 / 2 \pi$ radians, where a zero wavelength identifies oscillations that take place across the entire $2 \pi$ of the discharge channel in unison. The DFT is symmetric and redundant across the centerline of this matrix, as signals propagating, say, clockwise at $1 / 4$ revolution per frame are indistinguishable from those propagating counterclockwise at $3 / 4$ revolution per frame. Only a small section of the unique quadrant of the DFT is displayed here; the upper half of the frequency axis is truncated 

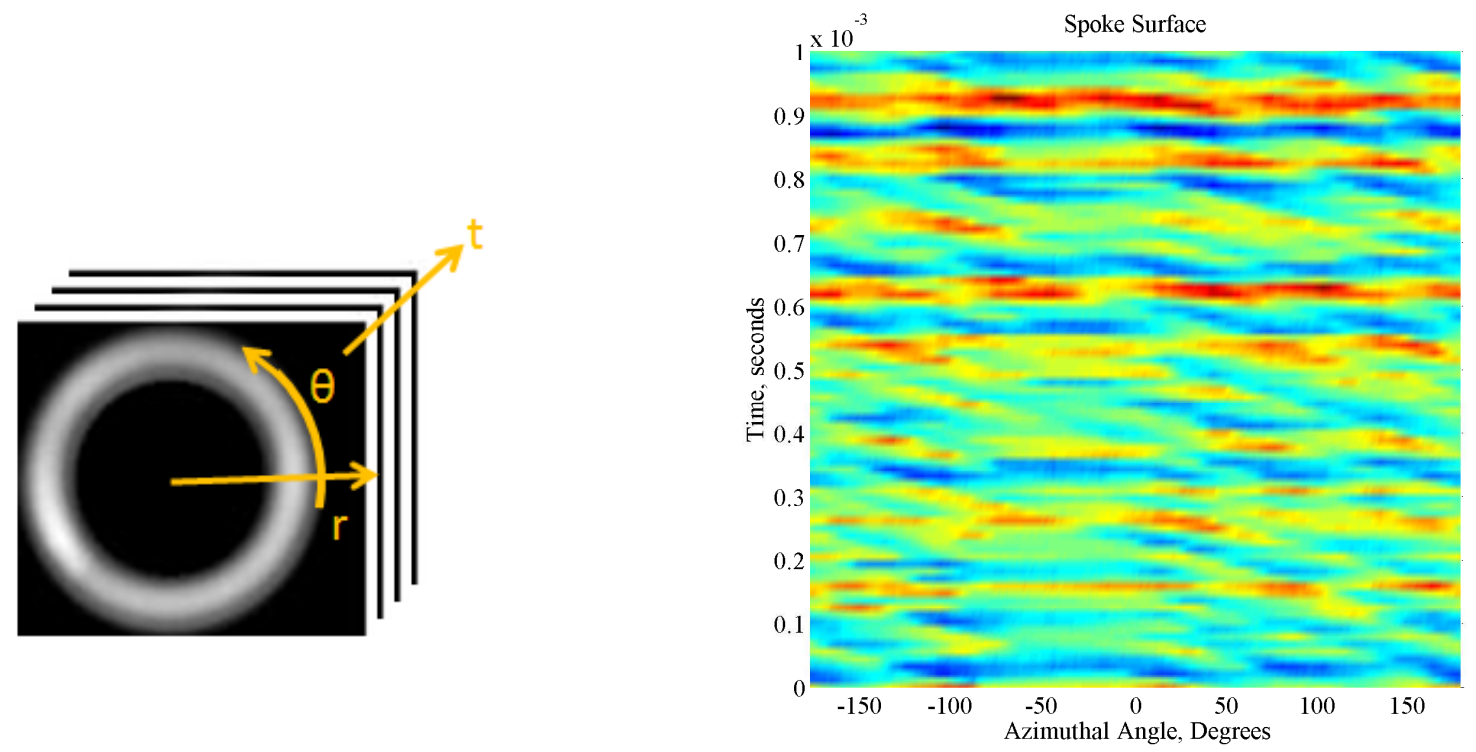

Figure 5. A video, represented as a 3D matrix of pixels in radius, angle and time, (left) is collapsed into a 2D spoke surface (right) by dividing the annular discharge channel into a number of discrete bins and averaging over the radial variation within each bin. The resulting spoke surface has a number of columns equal to the number of azimuthal bins and rows equal to the number of video frames. The parallel angled striations in the image correspond to spoke modes propagating in theta with time, while horizontal striations correspond to the breathing mode appearing in unison across the entire channel.

at the Nyquist frequency of the framerate, and the amplitudes of modes with wave numbers greater than about 8 are often negligibly small.
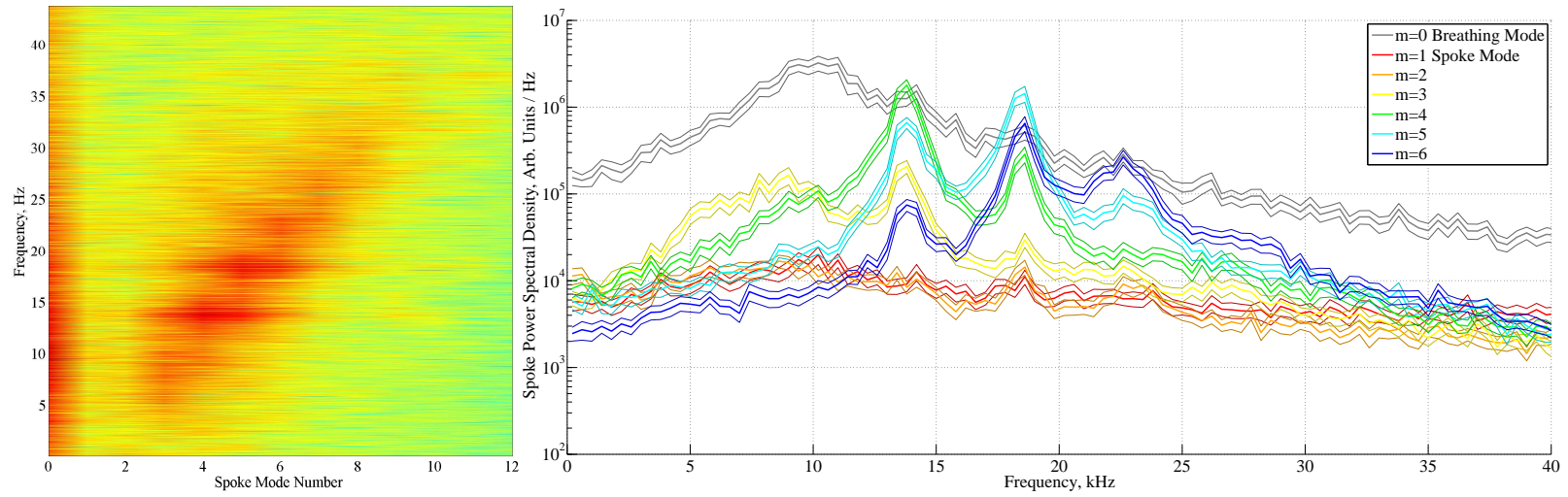

Figure 6. Left, the 2-D DFT of the spoke surface. Right, the strongest modes are broken out for 1-D representation. The $m=0$ mode is dominant and appears as a vertical red stripe along the $y$-axis in the 2-D DFT at left and in gray at right.

The final Fourier representation of the thruster video, at right in Figure 6, labels the discrete mode numbers as $m=0,1,2$ etc. These labels correspond to the number of simultaneous excitations around the channel at any one time:

$$
m \equiv k_{\theta} R=\frac{2 \pi R}{\lambda}
$$

where $k$ is the wavenumber and $R$ is the mean radius of the discharge channel. There is some smearing of a given mode's frequency peak into the other modes, but there is usually a clear dominant mode for any given peak. Here, the $m=0, m=4$ and $m=5$ modes are strongest.

Physically, these modes in the DFT correspond to the breathing and spoke modes. The $m=0$ mode is a unified oscillation across all 360 azimuthal degrees simultaneously, and is an excellent representation of the breathing mode 
and indeed the discharge current signal as a whole, matching the discharge current DFT almost exactly. The $m=1$ mode is a single pulse propagating azimuthally. The $m \geq 2$ modes have $m$ simultaneous spokes propagating around the channel. For all $m \geq 1$, the frequency peak $f_{m}$ of the $m^{\text {th }}$ mode identifies the linear velocity of spoke passage around the discharge channel as

$$
v_{m}=\frac{2 \pi R f_{m}}{m}
$$

For the $\mathrm{H6}$ a $m=1$ spoke mode with $f_{1}=1 \mathrm{kHz}$ travels at an approximate velocity $v_{1}=500 \mathrm{~m} / \mathrm{s}$. The $1 / \mathrm{m}$ term in the linear velocity equation is because the 2D DFT gives a local frequency at a fixed azimuthal location - if $m$ spokes are present, the local frequency is artificially boosted by a factor of $m$, so a $m=2$ spoke mode with $f_{2}=1 \mathrm{kHz}$ in the DFT only has a linear spoke velocity of $250 \mathrm{~m} / \mathrm{s}$, compared to $500 \mathrm{~m} / \mathrm{s}$ for the $m=1$ mode with the same frequency. Put another way, the $m=2$ mode with $f_{2}=1 \mathrm{kHz}$ is actually composed of two spokes, each propagating at $500 \mathrm{~Hz}$ but perfectly out of phase so that they combine to appear as a $1 \mathrm{kHz}$ wave passing any fixed observation point in the channel. An isolated probe in the channel cannot distinguish between modes with different global frequencies that manifest at the same local frequency, but high speed video of the full discharge channel can confidently distinguish between different spatial modes in the same frequency range.

In general it is not possible to use the raw pixel output of the camera directly as a measure of visible light intensity. Non-linearity of the bit depth of the camera sensor causes the pixel output to asymptote and saturate at high incident fluxes. This can be accounted for by calibration if necessary. However, for the particular cases of imaging the Hall thruster presented here, the high frame rates used are sufficient to keep the light intensity reaching the camera well inside the linear regime. The exception to this rule is the cathode, which is often an order of magnitude brighter than the discharge channel and is generally saturated in high speed video. Since the cathode portion of the frame is not used for analysis, this does not affect the results presented here.

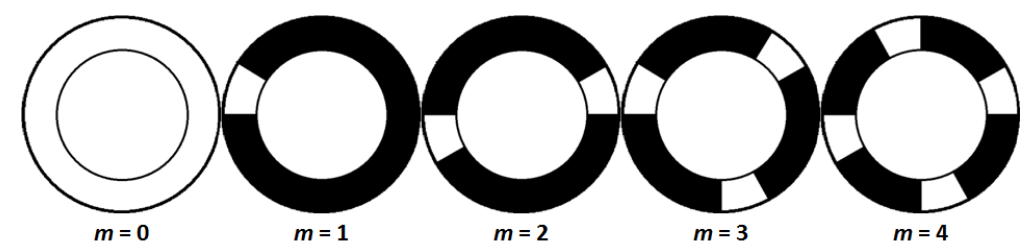

Figure 7. Physical meanings of the various spoke modes. White indicates luminous spokes propagating through an otherwise dark background. The mode number $m$ indicates the number of spokes simultaneously present. For $m \geq 1$, the spokes propagate azimuthally in the $E \times B$ direction. For $m=0$, the entire channel flashes in unison.

\section{B. Video postprocessing for visualization of rotating spokes}

The DFT method described above detects spoke modes well and quantifies their relative magnitude compared to the breathing mode, but it is sufficiently abstract that it becomes convenient to be develop a technique for visualizing the presence of spokes as well. In this section we will describe in detail how images like those in Figure 1 are generated. A step-by-step visual description of this process is shown in Figure 8.

Given the 3-D matrix of pixel intensities from Equation 6, we define a mean image $M$ as

$$
M(i, j)=\frac{1}{N_{k}} \sum_{k} p(i, j, k)
$$

where $M$ is a 2-D matrix of pixel intensities where each pixel's value is the average of all values measured by that pixel over the course of an entire video. Since the discharge current already gives a very good signal describing the $m=0$ breathing mode, it is not necessary to include it in a visualization. Indeed, it often forms a distraction that makes it more difficult to make out the spoke modes, especially if they are of small amplitude compared to the $m=0$ mode. To remove the breathing mode from the video without affecting visualization of the spoke modes as little as possible, the pixel intensity of the mean image is used to generate a set of normalizing factors that can be applied to the video to remove overall oscillations. This identically zeroes out the $m=0$ column of the 2D FFT while leaving the other modes untouched.

We define these normalizing factors $X$ as

$$
X_{k}=\sum_{i, j} M(i, j) / \sum_{i, j} p(i, j, k)
$$


where we recall that $k$ is the index of the video frame, so a unique normalizing factor is generated for each frame. This normalizing factor is multiplied across the corresponding raw video frame to brighten images that are dimmer overall than average and to dim images that are brighter overall than average, generating a new "normalized" video with a $m=0$ mode that is perfectly removed and normalized pixels with values given by

$$
p_{\text {norm }}(i, j, k)=X_{k} p(i, j, k)
$$

Raw images are shown in the first row of Figure 8, while in the second row the images are normalized. This has little apparent effect at this stage, but normalization greatly improves the clarity of the images in the later rows. After normalization the images go through several steps aimed at improving contrast. The cathode tends to be an order of magnitude brighter than the rest of the thruster, often saturating the image sensor and making it difficult to discern any detail in the channel. From row 2 to row 3 the cathode is cropped from the images. It is possible to manually crop a thruster image to remove the cathode and focus on the discharge channel, but we prefer to use an automated procedure and adopt the method of Kasa ${ }^{36}$, which is fast and relatively simple to implement. Using Kasa's leastsquares minimization method we fit a circle with the mean channel radius and define a channel width outside which all pixel values are set to zero. The Kasa fit is inaccurate when the whole channel is not captured in the image; for more robust circle-fitting algorithms we refer the reader to a comprehensive monograph on the subject by Chernov. ${ }^{37}$

Even without the cathode the contrast in the channel is still poor due to the large DC image signal, given by the mean image $M$. The spoke oscillations often appear with a pixel intensity amplitude $<10 \%$ of the mean pixel intensity, so AC-coupling is required to view them clearly. Thus, from row 3 to row 4 the pixel values are AC-coupled as

$$
p_{\text {norm }_{A C}}(i, j, k)=p_{\text {norm }}(i, j, k)-M(i, j)
$$

At this stage the spokes become clearly visible, though still in black and white. As a final step, since the naked eye perceives contrast in black and white rather poorly, the images are converted to false color from row 4 to row 5, where red indicates brighter regions than the mean image and blue indicates dimmer regions.

\section{Results}

Independent discharge current measurements from each anode segment were acquired at $1 \mathrm{MHz}$ over a duration of one second. FASTCAM video was also acquired for one second at 256 x 256 pixel resolution at 87,500 fps. While discharges at 105, 150 and $300 \mathrm{~V}$ were operated, only $300 \mathrm{~V}, 10 \mathrm{mg} / \mathrm{s}$ operation is examined in detail. The anode segments are numbered clockwise with segment 12 at the top as seen from the view of the thruster firing in Figure 3.

\section{A. Discharge Current Measurements to Individual Segments}

Time domain measurements show large amplitude oscillations that travel in the counterclockwise or $E \times B$ direction from segment to segment (Figure 9). Current deposition to each segment is not uniform, as the authors initially expected. Instead, some segments draw up to twice as much current on average as others. This may be due to several factors, including but not limited to: slight misalignment of the centrally mounted cathode, leading to preferential cathode coupling to some of the segments over the others; imperfections in the segmented anode mounting in the discharge channel, such that some segments sit farther forward axially and thus may "intercept" electrons that would otherwise have continued on to adjacent segments; slightly different bias voltages applied to each segment due to differences in voltage fall across the lead wires; or possibly outgassing or other effects linked to rapid data acquisition within a few minutes of thruster ignition before full bakeout was complete. The cathode orifice centerline sits slightly off thruster centerline due to imperfections in its construction and alignment, and the axial position of the segments varies by approximately $1.5 \mathrm{~mm}$ across all segments. As measured at room temperature in atmosphere the line resistance of each line varies by a few tenths of an ohm, which given the current levels on each segment should induce voltage variations only on the order of a few hundred millivolts. The discharge current was elevated to 10.9 amps at the time of data acquisition from the usual $9.2 \mathrm{amps}$ at this operating condition at steady state when using a contiguous anode.

While we note the nonuniformity of the discharge in the case of the segmented anode we hesitate to attribute it to any particular design defect. It is not clear that even in contiguous anode operation the discharge current is totally azimuthally uniform; instead this may be a feature of normal Hall thruster operation, even with the azimuthal symmetry of a center-mounted cathode. Both the mean DC discharge current levels of each segment and the amplitude of their AC oscillations, calculated as $\sqrt{2}$ times the root-mean-square of the AC-coupled signal, are shown in Table 1 and Figure 10. 

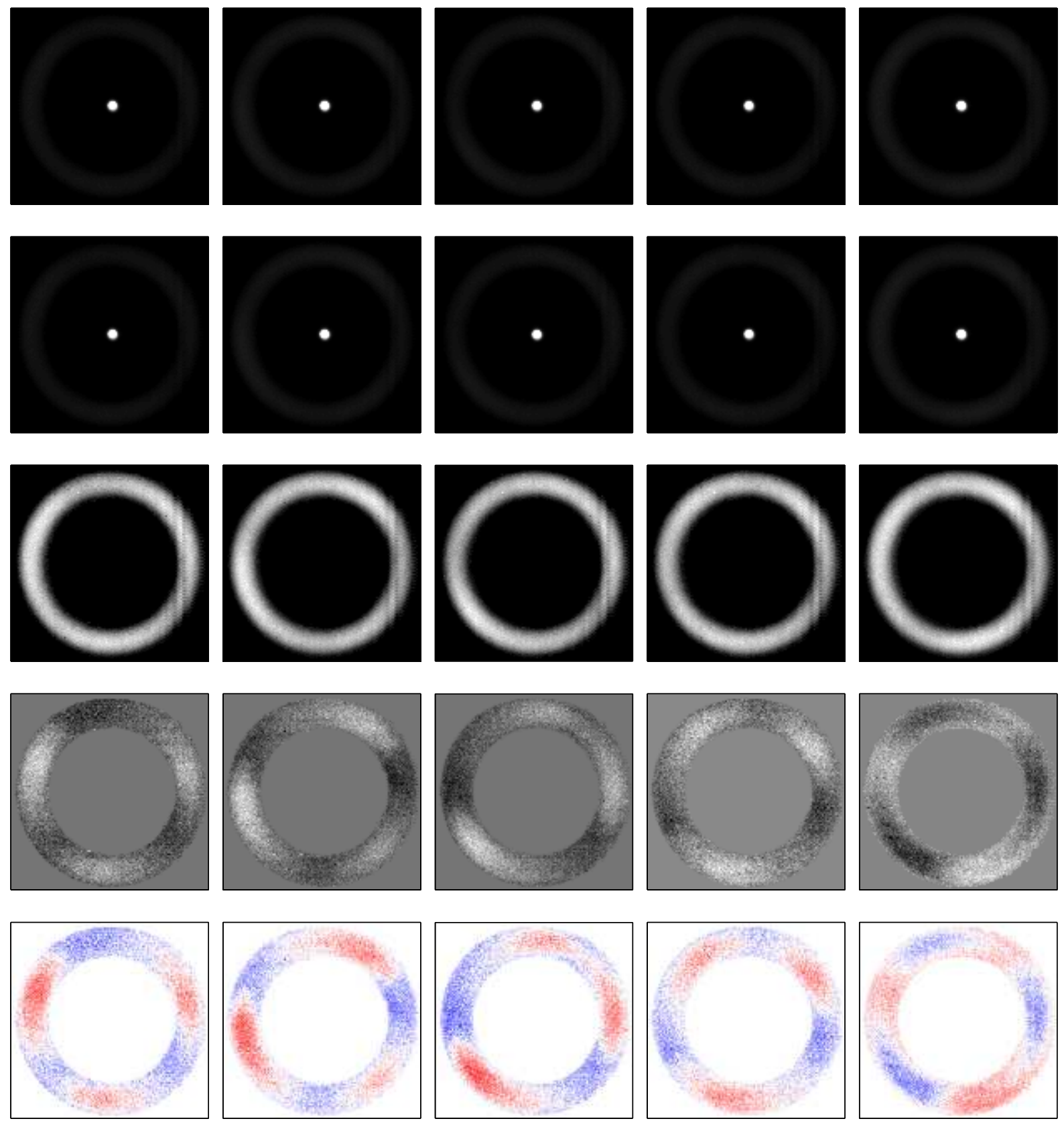

Figure 8. Sample images showing the high-speed video postprocessing technique used in this paper. In the top row, raw images of the H6 Hall thruster are in black and white with the central cathode as a very bright spot saturating the image. In the second row the images have been normalized relative to the mean image intensity over the whole video. In the third row the central cathode is cropped from the image, and in the fourth row the cropped image is AC-coupled by subtracting off the mean image. Both of these steps are aimed at improving contrast. Finally, the black and white AC-coupled image is converted to false color, where red indicates brighter and blue indicates dimmer regions than average. An $m=3$ spoke mode rotating counterclockwise becomes visible in the final rows. 


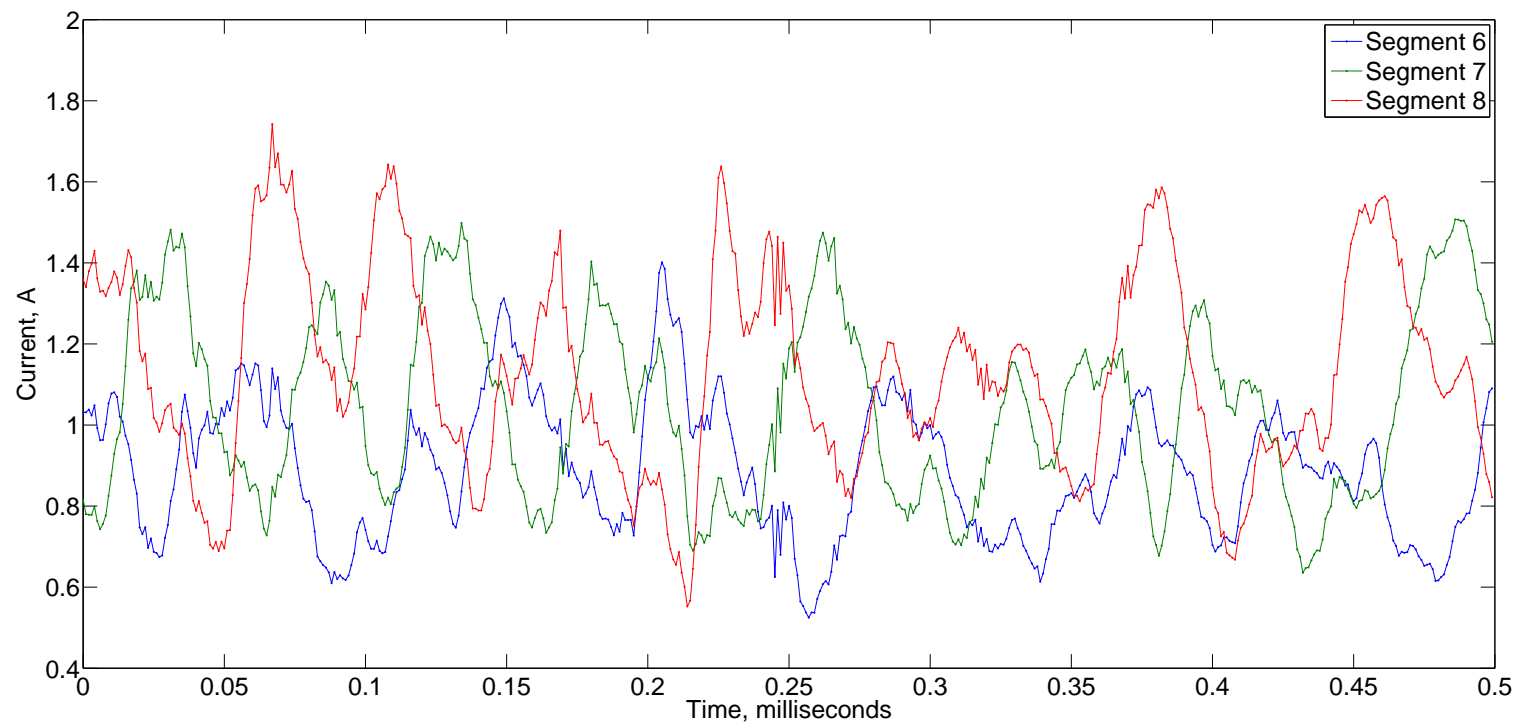

Figure 9. Current measured on three adjacent anode segments. The slight visible offset between signals indicates propagation of the wave structure, from segment 8 (red) toward segments 7 (green) and 6 (blue). Only three segments are shown for clarity, but all segments demonstrated large oscillations compared to the mean discharge current.

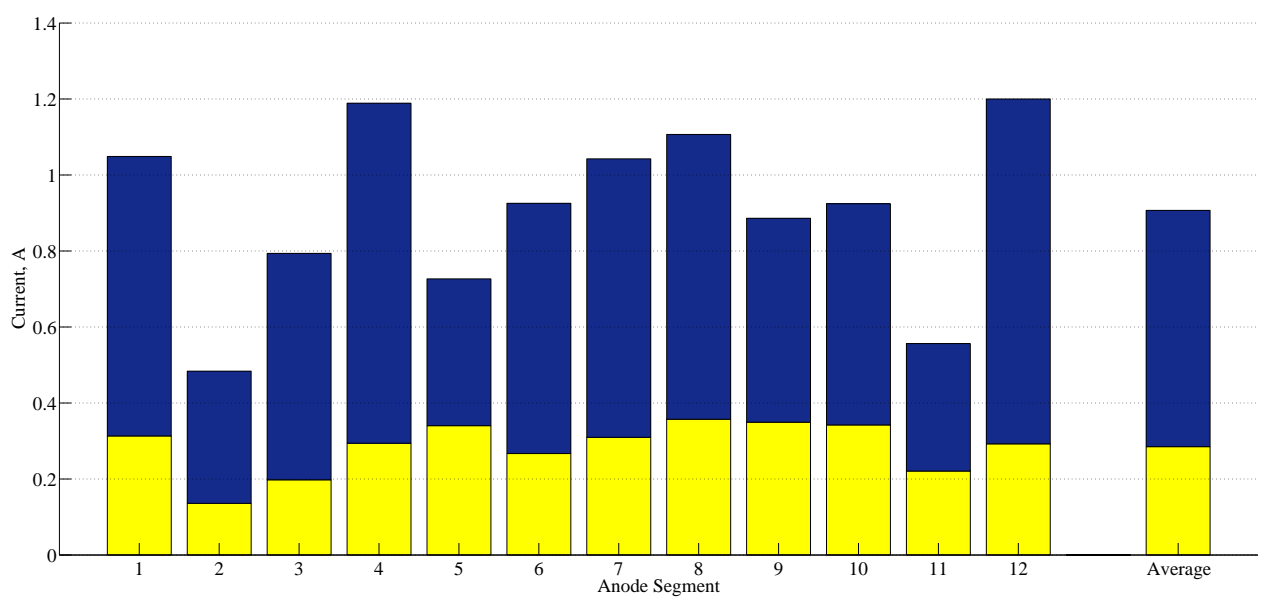

Figure 10. Mean discharge current (blue) and oscillation amplitudes (yellow) across the 12 anode segments.

\begin{tabular}{|c|c|c|c|}
\hline Anode Segment & DC Amplitude, A & AC Amplitude, A & AC/DC Ratio, \% \\
\hline \hline 1 & 1.05 & 0.31 & 30 \\
\hline 2 & 0.49 & 0.14 & 28 \\
\hline 3 & 0.79 & 0.20 & 25 \\
\hline 4 & 1.19 & 0.29 & 25 \\
\hline 5 & 0.73 & 0.34 & 47 \\
\hline 6 & 0.93 & 0.27 & 29 \\
\hline 7 & 1.04 & 0.31 & 30 \\
\hline 8 & 1.11 & 0.36 & 32 \\
\hline 9 & 0.89 & 0.35 & 39 \\
\hline 10 & 0.92 & 0.34 & 37 \\
\hline 11 & 0.56 & 0.22 & 40 \\
\hline 12 & 1.20 & 0.29 & 24 \\
\hline Average & $0.91 \mathrm{~A}$ & $0.29 \mathrm{~A}$ & $32 \%$ \\
\hline
\end{tabular}

Table 1. Mean discharge current, oscillation amplitude and their ratio across all 12 segments, tabulated from Figure 10. The cause of the spread in the DC amplitude is uncertain, but the ratio of oscillation amplitude to mean local discharge current stays fairly steady across these nonuniformities. 


\section{B. High-speed Video}

High-speed video of the thruster shows spokes rotating counterclockwise in the $E \times B$ direction. The sample postprocessed video stills in Fig. 11, generated as discussed in Section IV.B, show several spokes propagating in a $m=4$ mode. From Figure 12 the $m=4$ and $m=5$ spoke modes are dominant, though both are lower in the magnitude than the breathing mode. Recall that the postprocessing technique removes the $m=0$ mode from the images to make the higher spoke modes more visible. As the video DFT in Figure 12 shows, this is necessary because the $m=0$ peak is higher and the mode contains more power (the area under the spectral density plot) than all the other modes combined.

Figure 12 also shows that the spokes rotate at higher frequencies than the $m=0$ breathing mode oscillation. This is a feature unique to the segmented anode; in all videos of thrusters with a contiguous anode that the authors have previously viewed, the dominant spoke modes are at a lower frequency than the breathing mode. This will be addressed further in Section D. With the clear mode frequency breakdown from the FASTCAM DFT we can confidently identify the different modes present in the DFT of the discharge current.

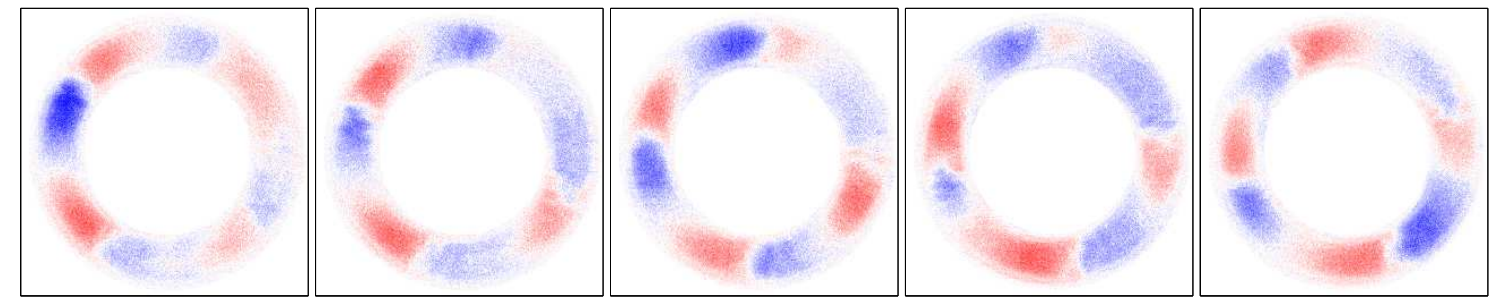

Figure 11. Postprocessed high speed video at 87,500 fps of the $\mathrm{H6}$ discharge channel operating at $300 \mathrm{~V}, 10 \mathrm{mg} / \mathrm{s}$ with the segmented anode. The $m=4$ mode is clearly visible and rotating in the $E \times B$ (counterclockwise) direction.

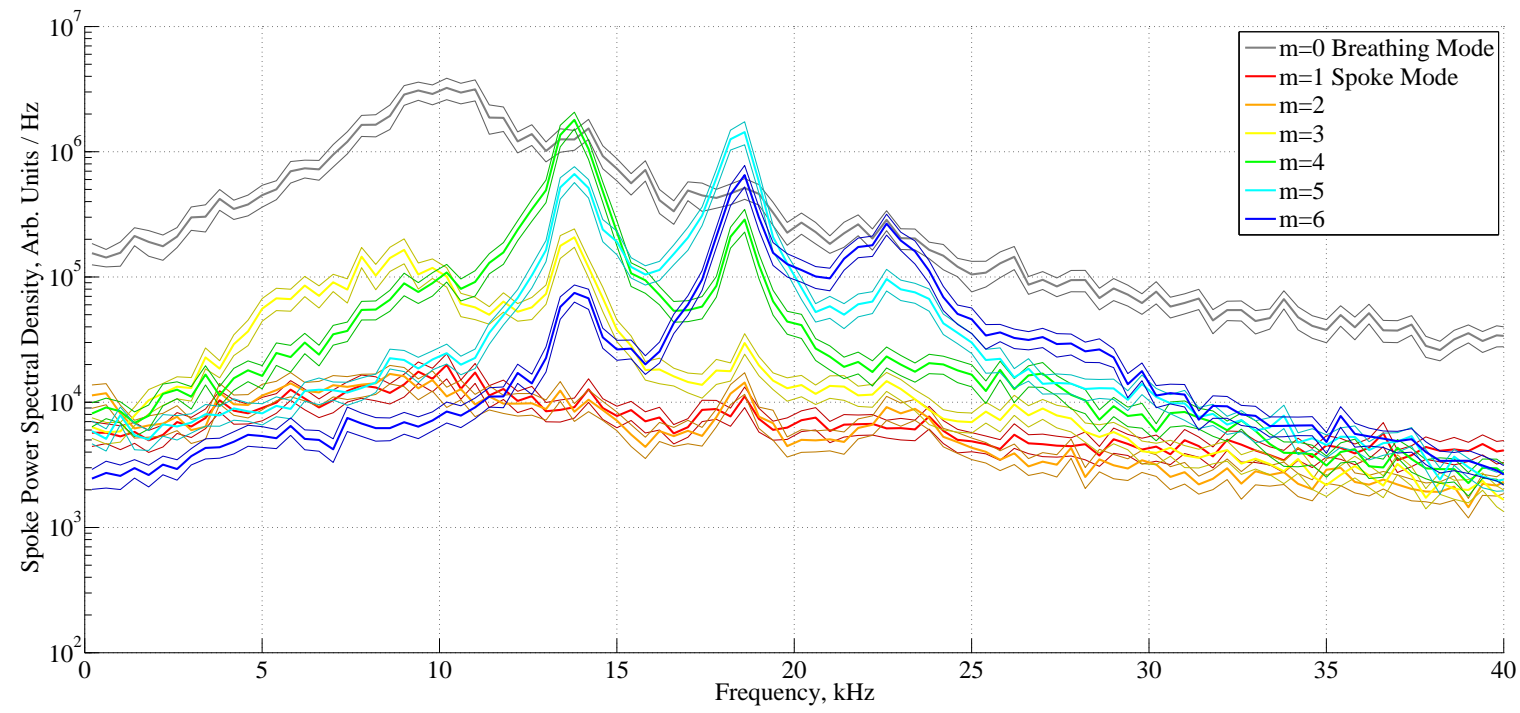

Figure 12. Selected modes from the high-speed video 2-D DFT showing the dominant oscillations present in the discharge. The $m=0$ breathing mode is dominant, but the $m=4$ and $m=5$ modes are close behind. Some smearing of the dominant mode peaks into adjacent numbers is inevitable, but the largest amplitude peak is the dominant mode at each frequency, while the smearing is an artifact of the turbulence of the discharge.

\section{Relation between individual segment discharge currents and total discharge current}

One likely reason spoke modes go so often undetected in Hall thrusters, despite their near universal presence, is their concealment in total discharge current measurements. Individual segment measurements show strong peaks at 14 and $18 \mathrm{kHz}$, which from the previous section we may confidently identify as the $m=4$ and $m=5$ spoke modes. 
However, as the current from more segments is considered in aggregate (see Figure 13), the relative dominance of the spoke modes in the power spectrum diminishes in comparison to the more broadly humped $m=0$ breathing mode at $9 \mathrm{kHz}$.

The physical basis of this concealment when more segments are considered together is that the breathing mode appears weakly but in unison across all segments, while the spokes are strong but relatively out of phase from one segment to another. As the currents from more segments are added together, the contributions from the breathing mode add constructively while those from the spoke modes add destructively. The result is an aggregate signal that downplays the presence of spoke modes in the discharge by effectively integrating away the azimuthal spoke mode over the full $2 \pi$ radians of the complete set of anode segments.

The power spectrum measured from the Tektronix split-core Hall probes on the anode line confirm that the summation of the currents from the NT-5 sensors on each segment give an accurate picture of the total discharge current oscillations.

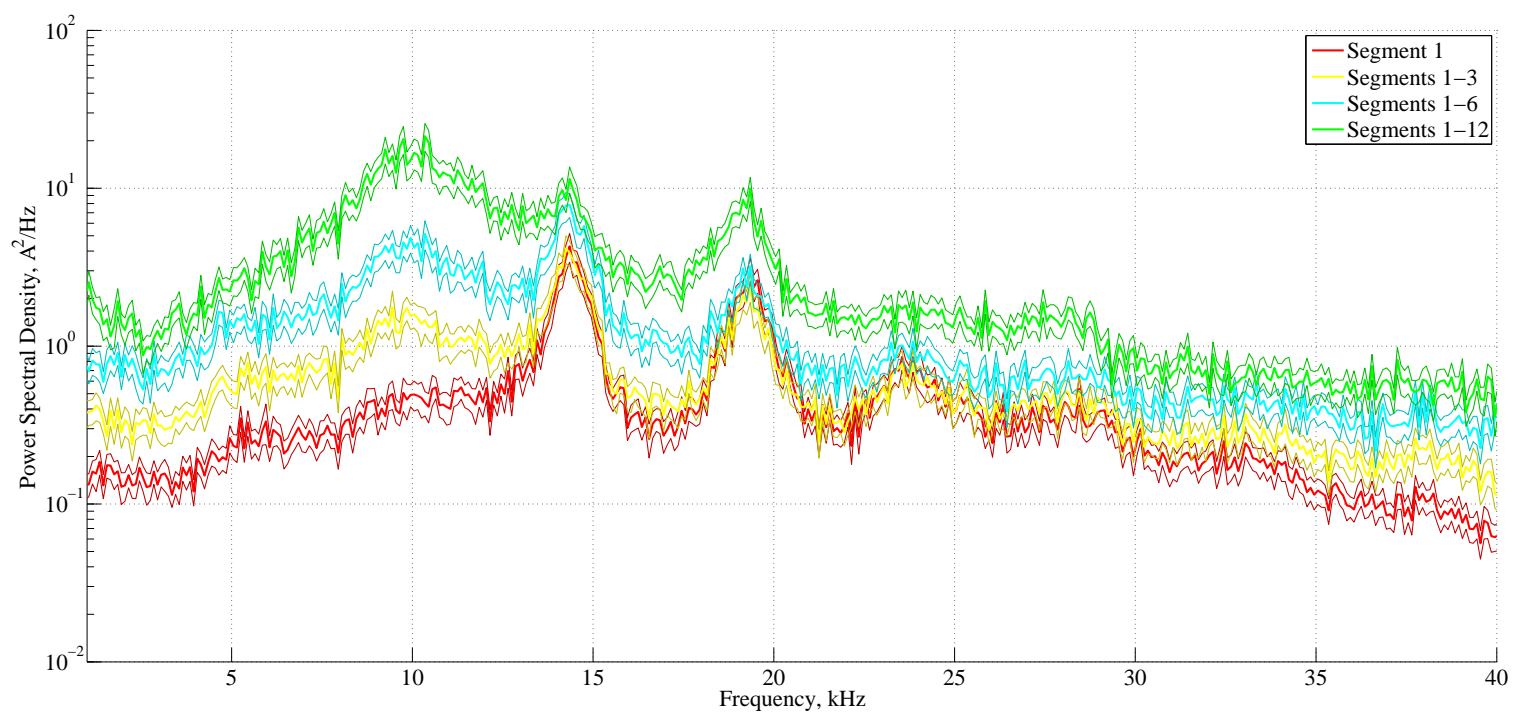

Figure 13. Segmented anode discharge current DFTs for individual and groups of segments. The DFT of a single segment (red) shows strong peaks corresponding to the $m=4$ and $m=5$ spoke modes shown in Figure 12, with little evidence of the $m=0$ breathing mode at $9 \mathrm{kHz}$. However, as more segments are considered together (yellow, blue) the breathing mode grows while the spoke modes diminish in relative magnitude. Finally, when all 12 segments are combined to give the entire thruster discharge current (green) the spoke modes drop below the breathing mode. In conventional contiguous anode thrusters the spoke modes are not even visible, and it is likely that only the discrete nature of the segmented anode, chopping the electron current as it passes between segments, preserves their visibility in the total discharge current spectrum seen here.

\section{Comparison to nominal operation without segmented anode}

The segmented anode operates in a considerably different mode than the contiguous anode even at identical discharge voltage, mass flow rate and magnet settings. Because of the quasi-pulsed operation of the H6 in this test to preserve electrical isolation of the segmented anode, it is difficult to say what portion of these differences are attributable to changes in the thruster geometry and electrode configuration vs. due to the outgassing from the discharge channel walls and other startup transients. The segmented anode was also fired with the cathode keeper on and drawing $1.7 \mathrm{~A}$ of current as a precaution in case the thruster went unstable.

\begin{tabular}{|c|c|c|}
\hline & Contiguous Anode & Segmented Anode \\
\hline \hline$\overline{I_{D}}$ & $9.2 \mathrm{~A}$ & $10.9 \mathrm{~A}$ \\
\hline$\widetilde{I_{D}}$ & $3.8 \mathrm{~A}$ & $0.9 \mathrm{~A}$ \\
\hline Dominant spoke mode & $m=2,3$ & $m=4,5$ \\
\hline Cathode keeper on? & No & Yes \\
\hline Spoke frequencies visible in total $I_{D}$ spectrum? & No & Yes \\
\hline
\end{tabular}

Table 2. Key feature comparison, segmented anode vs. contiguous anode operation at $300 \mathrm{~V}, 10 \mathrm{mg} / \mathrm{s}$ 

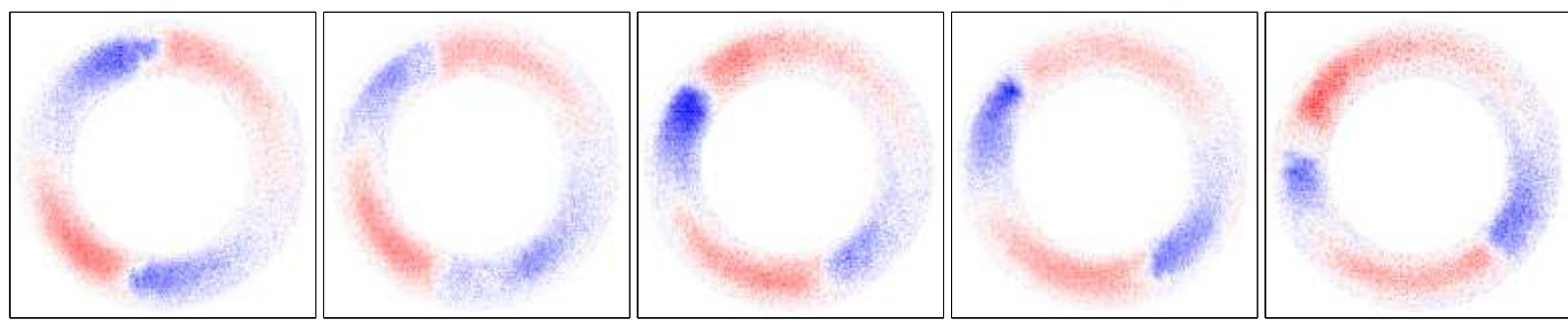

Figure 14. Postprocessed high speed images of the $\mathrm{H6}$ operating at a matching $300 \mathrm{~V}, 10 \mathrm{mg} / \mathrm{s}$ operating condition with the original contiguous anode. The $m=2$ spoke mode is clearly visible. This video was taken at $162,750 \mathrm{fps}$, but only every fourth frame is shown to make the rotation of the spoke more apparent since the spoke velocity is much lower with the contiguous anode.

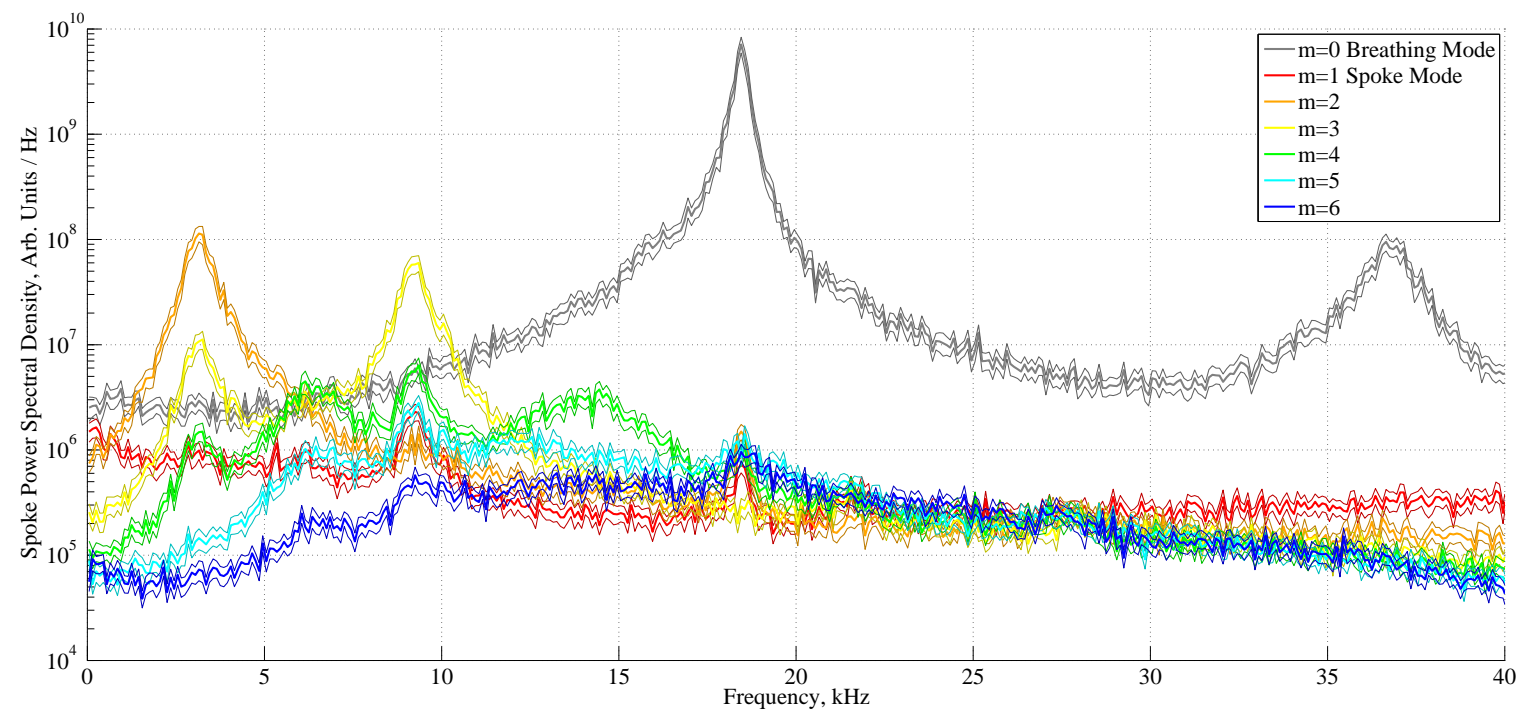

Figure 15. Dominant modes of the 2D DFT of the spoke surface for the $\mathrm{H6}$ operating at $300 \mathrm{~V}, 10 \mathrm{mg} / \mathrm{s}$ with the original contiguous anode. The $m=2$ and $m=3$ modes are the most prominent spoke modes, but the breathing mode is exceptionally strong at this condition and its second harmonic at $37 \mathrm{kHz}$ is visible as well. The low frequencies of the spoke modes here are typical; the segmented anode is the only thruster configuration yet observed with spoke frequencies higher than the breathing mode frequency.

Comparison of the discharge current oscillations and FASTCAM videos show that the introduction of the segmented anode shifts spoke mode oscillations to higher mode numbers than in contiguous anode operation (see Figures 12 and 15). The $m=2$ and $m=3$ spoke modes are strongest in the contiguous anode configuration, though both are dwarfed by the dominant breathing mode. The postprocessed FASTCAM video frames of Figure 14 show the $m=2$ mode propagating in the $E \times B$ direction around the channel. If the magnitude of the global discharge current oscillations $\widetilde{I}_{D}$ are taken as a measure of stability, the segmented anode was actually more stable than the contiguous anode - during segmented anode operation these oscillations dropped in spite of an increased overall discharge current (Table 2).

Figure 15 also shows that the dominant spoke mode frequencies with the contiguous anode all lie below the breathing mode frequency, which is typical of all other high speed Hall thruster videos we have acquired. The H6 with segmented anode is the only Hall thruster configuration we have observed with spoke modes with higher frequencies than the breathing mode. It is also the only configuration where the spoke modes show up in the DFT of the total discharge current. We attribute both of these effects to the introduction of the 12 discrete boron nitride spacers that effectively chop the discharge current and seed spoke modes at higher mode numbers and frequencies than are normally stable. For example, the $m=4$ spoke mode at $14 \mathrm{kHz}$ is barely noticeable in Figure 15, while it is dominant in Figure 12. In the contiguous anode the electron current can deposit smoothly around the azimuth of the anode without disruption and modes higher than $m=3$ are rarely observed.

While there are clear changes that the segmented anode introduces to the discharge, the previous sections also make clear that the visible oscillations detected by high speed camera are real plasma behavior associated with cross- 
field electron current. The primary value of this work lies less in whether the segmented anode preserves the discharge characteristics of contiguous anode and more in conclusively demonstrating that visible rotating spokes detected via high speed camera correspond to azimuthally localized electron current deposition to the anode. Given the excellent matching between FASTCAM video spoke frequencies and local current oscillation frequencies on individual segments, this point is established.

\section{Calculation of Cross-field Electron Current due to Rotating Spokes}

Given the amplitude of the oscillations at the anode it is possible to estimate the magnitude of oscillations in the plasma density and potential and ultimately determine the current attributable to the rotating spokes. We assume the oscillations in density and electric field are either in phase or approximately in phase with a phase shift $\delta$ such that $\cos (\delta) \approx 1$. While in situ measurements of these quantities were not made, this assumption is consistent with the measurements made by Janes and Lomas. Recall from Eqn. 5 that

$$
\begin{gathered}
j_{e z}(\theta)=\frac{q \pi}{4 B} E_{z}\left(n^{\prime} \sin (\theta)+\frac{\left(n^{\prime}\right)^{2}}{n_{0}} \sin ^{2}(\theta)\right) \\
\overline{j_{e z}}=\frac{q \pi}{8} \frac{E_{z}}{B} \frac{\left(n^{\prime}\right)^{2}}{n_{0}}
\end{gathered}
$$

The derivation of Equation 3 by Yoshikawa assumed that $n^{\prime} \ll n_{0}$. However, we note that Janes found excellent agreement with this expression even with $n^{\prime} / n_{0}=0.5$. Following the small perturbation assumption, we approximate

$$
j_{e z}(\theta) \simeq \frac{q \pi}{4 B} E_{z} n^{\prime} \sin (\theta)=\widetilde{j_{e z}} \sin (\theta)
$$

where

$$
\widetilde{j_{e z}} \equiv \frac{q \pi}{4} \frac{E_{z}}{B} n^{\prime}
$$

Of the quantities in Equation 14, $\widetilde{j_{e z}}$ can be calculated from the segmented anode, the electric field $E_{z}$ is known from internal plasma potential measurements in the $\mathrm{H} 6$ discharge channel by $\mathrm{Reid}^{32}$, and the magnetic field magnitude $B$ is readily obtained from finite element simulations in Infolytica's MagNet software. With these values in hand one can calculate the density perturbation and, finally, the total cross-field electron current $\overline{j_{e z}}$.

The current signal on each anode segment is due to a combination of the breathing and spoke modes, but $\widetilde{j_{e z}}$ is the oscillation amplitude due to the spoke modes only. Fourier analysis of the current to the anode segments in the previous section showed that the breathing mode $m=0$ oscillation occurs in phase across the whole channel, while the spokes are in general not in phase. To this end, we attempt to isolate the current oscillations due to the spoke modes from the breathing mode by a similar technique to the one outlined in Section IV.B to aid in spoke visualization, essentially treating the segmented anode as an effective 12-pixel image. We define a series of normalizing factors $X_{t}$ defined at each moment of data acquisition as the ratio of the mean discharge current $\overline{I_{D}}$ to the instantaneous discharge current $I_{t}$

$$
X_{t} \equiv \overline{I_{D}} / I_{t}
$$

and apply this normalization to each segment to scale away the $m=0$ oscillations and isolate the spokes more fully. This method serves well by visual inspection in video postprocessing, and it makes a small correction to the segmented anode current measurements, on average about a $4 \%$ reduction in oscillation amplitude. Considering the small amplitude of the breathing mode compared to the spoke mode on the individual segments as shown in the single segment DFT in Figure 13, this fraction seems reasonable. With the normalizing correction applied, the magnitude of the spoke oscillations is again assumed to be $\sqrt{2}$ times the RMS value of the normalized current oscillations, shown in Table 3.

Knowing the area of each anode segment, approximately $8.6 \mathrm{~cm}^{2}$, the average normalized current oscillation 0.27 A above gives a spoke current density oscillation amplitude $\widetilde{j_{e z}}=32 \mathrm{~mA} / \mathrm{cm}^{2}$. On channel centerline from the anode surface up to approximately $75 \%$ of depth of the discharge channel where the ionization and acceleration regions begin, an approximate average for $E_{z} / B$ is $10^{4} \mathrm{~m} / \mathrm{s}$, where given the high degree of field symmetry along channel centerline we take $B=B_{r}$. From Equation 14, this gives a perturbation density $n^{\prime}=2.5 \times 10^{11} \mathrm{~cm}^{-3}$. In the near-anode 


\begin{tabular}{|c|c|c|c|}
\hline Segment Index & $\sqrt{2}$ (RMS), Amps & $\sqrt{2}$ (RMS) Normalized, Amps & \% Change \\
\hline \hline 1 & 0.313 & 0.306 & -2.3 \\
\hline 2 & 0.140 & 0.140 & 0.0 \\
\hline 3 & 0.198 & 0.177 & -10.6 \\
\hline 4 & 0.294 & 0.289 & -1.9 \\
\hline 5 & 0.340 & 0.337 & -1.0 \\
\hline 6 & 0.267 & 0.250 & -6.2 \\
\hline 7 & 0.310 & 0.292 & -5.8 \\
\hline 8 & 0.357 & 0.348 & -2.6 \\
\hline 9 & 0.350 & 0.342 & -2.3 \\
\hline 10 & 0.342 & 0.319 & -6.9 \\
\hline 11 & 0.221 & 0.211 & -4.4 \\
\hline 12 & 0.293 & 0.288 & -1.6 \\
\hline Average & 0.285 & 0.274 & $-3.8 \%$ \\
\hline
\end{tabular}

Table 3. Anode segment currents after normalization of the breathing mode. The correction is small, about $4 \%$ on each segment, in line with the small spectral density of the breathing mode compared to the spoke modes shown on the single segment current DFT in Figure 13.

region the neutral density, based on the blended calculations between OML and thin-sheath Langmuir probe regimes of $\operatorname{Reid}^{33}$, is $n_{0}=2.5 \times 10^{11} \mathrm{~cm}^{-3}$. This means that the calculated density perturbation from the segmented anode currents is of the same order as the mean background density from the time-averaged measurements of Reid, i.e., $n^{\prime} / n_{0}=1$. We will further quantify the effects of the approximation $n^{\prime} / n_{0} \ll 1$ from Equation 13 in Section VI.A, but we pause here to note that even for $n^{\prime} / n_{0}$ of order unity the correction is small.

With the magnitude of the density perturbation in hand, the total cross-field electron current may be calculated from Equation 12. Using the above-mentioned values for $n_{0}$ and $E_{z} / B$ in the rear of the discharge channel gives a spoke-induced axial current density of

$$
\begin{aligned}
& \overline{j_{e z}}=\frac{q \pi}{8} \frac{E_{z}}{B} \frac{\left(n^{\prime}\right)^{2}}{n_{0}} \\
& \overline{j_{e z}}=50 \mathrm{~mA} / \mathrm{cm}^{2}
\end{aligned}
$$

Over 12 anode segments this current density corresponds to a total spoke-induced current of $5.2 \mathrm{amps}$ in a total discharge of 10.9 amps. Physically this means that at the anode, or in the near-anode region, about half of the discharge current is carried by rotating spokes.

\section{A. Sources of error}

At least three sources of error are likely to affect the accuracy of the calculation of cross-field electron current above. The first and most easily quantified is the assumption in Equation 13 that $n^{\prime} / n_{0} \ll 1$, when our estimates above place it near unity. We set aside any effects of this discrepancy on Yoshikawa's derivation of Equation 3, in light of the good agreement Janes found with the theory even for $n^{\prime} / n_{0}=0.5$, and focus on errors in the approximation of Equation 13. If we denote the ratio of densities $n^{\prime} / n_{0}$ by $\epsilon$ and express Equation 4 as

$$
j_{e z}(\theta, \epsilon)=\widetilde{j_{e z}}\left(\sin (\theta)+\epsilon \sin ^{2}(\theta)\right)
$$

it becomes clear that the error in the calculation of $n^{\prime}$ from $\widetilde{j_{e z}}$ in Equation 13 is due to the error in approximating the amplitude of

$$
f(\theta, \epsilon)=\left(\sin (\theta)+\epsilon \sin ^{2}(\theta)\right)
$$

as identical to the amplitude of $\sin (\theta)$ alone. A bit of calculus shows that the amplitude of $f(\theta, \epsilon)$ is given by

$$
\widetilde{f}(\theta, \epsilon)= \begin{cases}1, & \epsilon<1 / 2 \\ \frac{1}{2}\left(1+\epsilon+\frac{1}{4 \epsilon}\right), & \epsilon>1 / 2\end{cases}
$$


where for $\epsilon \leq 1 / 2$ the $\sin ^{2}(\theta)$ perturbation raises both the peak and trough of the sine wave by the same amount, leaving the amplitude unaffected. If we denote the zeroth order approximation $\epsilon=1$ from the values in the previous section as $\epsilon_{0}$ (not to be confused with the vacuum permittivity) then successive approximations yield

$$
\begin{aligned}
& \tilde{f}(\theta, 1)=9 / 8 \widetilde{f}(\theta, 0) \rightarrow \epsilon_{1}=\frac{8}{9} \epsilon_{0}=0.889 \\
& \widetilde{f}(\theta, 8 / 9)=65 / 64 \widetilde{f}(\theta, 1) \rightarrow \epsilon_{2}=\frac{64}{65} \epsilon_{1}=0.875
\end{aligned}
$$

and further corrections to $\epsilon$ are negligibly small. The error in calculation of the mean cross-field current from Equation 12 is proportional to the square of this correction factor as

$$
\overline{j_{e z}}=\frac{q \pi}{8} \frac{E_{z}}{B} \frac{\left(n^{\prime}\right)^{2}}{n_{0}}=\frac{q \pi}{8} \frac{E_{z}}{B} \epsilon_{2}\left(\epsilon_{2} n_{0}\right)=\epsilon_{2}^{2}\left(50 \mathrm{~mA} / \mathrm{cm}^{2}\right)=38 \mathrm{~mA} / \mathrm{cm}^{2}
$$

for a potential reduction in current density of $23 \%$.

The second source of error is in the estimate of the plasma density and potential from the internal measurements of Reid. Setting aside possible differences in H6 plasma structure between the contiguous and segmented anode, both the Langmuir and floating emissive probes are subject to substantial errors on their own, which Reid notes as $>50 \%$ for the Langmuir probe plasma density measurements. Taking this $50 \%$ factor into account, the range of current densities extends from 19 to $57 \mathrm{~mA} / \mathrm{cm}^{2}$, for a total current of between 2 and 6 amps.

The third source of error is due to our poor understanding of the theory of rotating spoke formation, in particular whether spokes are most sensitive to plasma parameters deep in the near-anode region of the discharge channel, or else to values near the exit plane or perhaps in the near-field plume. For simplicity's sake we have here used the density in the near-anode region and approximated the ratio $E_{z} / B$ by a value that is reasonable over much of the discharge channel, but given the rapid axial transit times of the electrons it may be that plasma parameters farther downstream are more appropriate. Consider that, for $\epsilon \sim 1$ the azimuthal field $E_{\theta}$ is of the order of $E_{z}$ from Equation 3, meaning the typical electron may pass axially along the spoke through the few centimeters of discharge channel length in a matter of microseconds. While it is impossible to quantify this manner of error, it again motivates deeper investigation of the rotating spoke instability.

\section{Conclusions}

In spite of a vast body of literature on potential mechanisms for anomalous electron transport in Hall thrusters, quantitative assessments of the contributions of these mechanisms to the total cross-field current are rare. The lack of clear measurements of whether collisional or turbulent transport mechanisms are dominant, and at a finer level the type and location of collisions or the frequency range of most interest for turbulence, hampers efforts to predictively model the the Hall thruster plasma even now, some 40 years after the first device entered orbit.

This work presents the first experimental measurements of the cross-field current due to rotating spokes in a modern high-power Hall thruster, and moreover demonstrates that these spokes carry a substantial portion of the total discharge current, on the order of 50\%, in the near-anode region. In spite of the large uncertainty associated with isolating the cross-field current due to spokes, this level of transport is consistent with the much higher current density device of Lomas and the smaller, low-power device of Ellison. It is remarkable that these spokes are still an influential electron transport mechanism in light of the substantial evolution of Hall thruster design since the 1960s when they were first detected. The large role the spoke instability plays across widely different operating regimes and thruster geometries indicates a robust formation mechanism in crossed field devices, and one that merits further study.

Discharge current spectra from the H6 Hall thruster with the segmented anode match the frequencies observed in DFTs of high-speed video of the Hall thruster discharge channel, demonstrating that the visible rotating spoke modes observed in video are the same ones responsible for passing electron current to the anode. Individual segment current measurements also show that the spoke modes are the dominant electron current oscillation near the anode, and that the apparent dominance of the breathing mode in conventional discharge current measurements is merely due do the effective integration of such measurements over the full $2 \pi$ of the discharge channel, washing away the azimuthal variations of the spokes. 


\section{Future Work}

While the segmented anode demonstrates the leading role that rotating spokes play in electron transport in the near-anode region, it is less clear how far downstream in the channel and into the plume this role extends. Careful processing of some high speed videos reveal faint traces of spokes over the inner pole, azimuthally offset from the spokes in the channel. These hints of more extensive spoke behavior, along with recent time-resolved plume plasma potential measurements by $\mathrm{Smith}^{38}$, suggest that the rotating spokes may extend out into the plume in some sort of helical structure and bridge the cathode to the anode. Future work on electron transport in Hall thrusters must address the near-field plume and describe the mechanisms that couple the cathode to the exit plane of the Hall thruster. The rotating spokes discussed in this paper appear to be a promising mechanism to tackle first.

In our opinion, the most likely method to achieve this goal is through time-resolved measurements of plasma potential and density throughout the near-field, with synchronous high speed video and discharge current measurements. Lobbia has pioneered a technique using the Hall thruster discharge current as a carrier signal to stitch together temporally and spatially disparate high-speed Langmuir probe measurements into a single time-resolved 2-dimensional plume map in $r$ and $z$, demonstrating convincing reconstructions of the temporal evolution of the Hall thruster breathing mode. ${ }^{39}$ High speed video may provide a second carrier signal to extend a time-resolved plume map into $\theta$ in the same fashion, permitting direct evaluation of the cross-field current throughout the Hall thruster plume in full 3-D.

\section{Acknowledgments}

The Photron SA5 FASTCAM was made available through the support of the Michigan/AFRL Center of Excellence in Electric Propulsion (MACEEP), grant FA9550-09-1-0695, under joint program managers Dr. Mitat Birkan (AFOSR) and Dr. James Haas (AFRL/RZ). The data analysis presented in this paper would not have been possible without computing resources funded by a Strategic University Research Partnership (SURP) with the Jet Propulsion Laboratory under principal investigator Dr. Richard Hofer. The first author is grateful for the support of the National Science Foundation Graduate Research Fellowship Program, as well as the Tau Beta Pi Foundation and the National Defense Industry Association. Finally, the authors thank Dr. Justin Koo of the Air Force Research Laboratory at Edwards Air Force Base for the idea to build a segmented anode, and master machinist Terry Larrow of the University of Michigan Department of Aerospace Engineering, without whose expertise that idea could never have left the drawing board.

\section{References}

${ }^{1}$ Zhurin, V. V., Kaufman, H. R., and Robinson, R. S., "Physics of closed drift thrusters," Plasma Sources Sci. Technol, Vol. 8, No. 1, 1999.

${ }^{2}$ Arkhipov, B. A., Vinogradov, V. N., Kozubsky, K. N., Kudryavtsev, S. S., Maslennikov, N. A., and Murashko, V. M., "Development and application of electric thrusters at EDB Fakel," 25th International Electric Propulsion Conference, IEPC-97-004, 1997.

${ }^{3}$ Chen, F. F., Introduction to Plasma Physics and Controlled Fusion, Plenum Press, New York, 1984.

${ }^{4}$ Janes, G. S. and Lowder, R. S., "Anomalous Electron Diffusion and Ion Acceleration in a Low-Density Plasma," Physics of Fluids, Vol. 9 , 1966, pp. 1115.

${ }^{5}$ McDonald, M. S. and Gallimore, A. D., "Rotating Spoke Instabilities in Hall Thrusters," IEEE Transactions on Plasma Science, 2011, (to be published).

${ }^{6}$ Parker, J. B., Raitses, Y., and Fisch, N. J., "Transition in electron transport in a cylindrical Hall thruster," Applied Physics Letters, Vol. 97, No. 9, 2010, pp. 091501.

${ }^{7}$ Ellison, L., Raitses, Y., and Fisch, N. J., "Cross-field Current through a Rotating Spoke," 53rd Annual Meeting of the American Physical Society Division of Plasma Physics, Poster Presentation, 2010.

${ }^{8}$ Ellison, L., Raitses, Y., and Fisch, N. J., "Fast Camera Imaging of Hall Thruster Ignition," IEEE Transactions on Plasma Science, 2011, (to be published).

${ }^{9}$ Lobbia, R. B. and Gallimore, A. D., "Performance Measurements from a Cluster of Four Hall Thrusters," 30th International Electric Propulsion Conference, IEPC 2007-177, 2007.

${ }^{10}$ Martinez, R., Hoskins, A., Peterson, P., and Massey, D., "Development status of the helicon Hall thruster," 31st International Electric Propulsion Conference, IEPC 2009-120, 2009.

${ }^{11}$ Hofer, R. R., Development and Characterization of High-Efficiency, High-Specific Impulse Xenon Hall Thrusters, Ph.D. thesis, University of Michigan, 2004.

${ }^{12}$ Liang, R. and Gallimore, A., "Far-Field Plume Measurements of a Nested-Channel Hall-Effect Thruster," 49th AIAA Aerospace Sciences Meeting, AIAA-2011-1016, 2011.

${ }^{13}$ Lomas, P. J. and Kilkenny, J. D., "Electrothermal instabilities in a Hall accelerator," Plasma Physics, Vol. 19, No. 4, 1977, pp. $329-341$.

${ }^{14}$ Morozov, A. I., “The conceptual development of stationary plasma thrusters," Plasma Physics Reports, Vol. 29, No. 3, 2003 , pp. $235-250$. 
${ }^{15}$ Bugrova, A. I., Morozov, A. I., and Kharchevnikov, V. K., "Experimental study on Near Wall Conductivity," Fiz. Plazmy, Vol. 16, 1992, pp. 849 .

${ }^{16}$ Morozov, A. I. and Savel'ev, V. V., “Theory of the Near-Wall Conductivity,” Plasma Physics Reports, Vol. 27, No. 7, 2001, pp. 570.

${ }^{17}$ Smith, A. W. and Cappelli, M. A., "Single particle simulations of electron transport in the near-field of Hall thrusters," Journal of Physics D: Applied Physics, Vol. 43, No. 4, 2010, pp. 045203.

${ }^{18}$ Litvak, A. A. and Fisch, N. J., "Resistive instabilities in Hall current plasma discharge," Physics of Plasmas, Vol. 8, No. 2, Feb. 2001, pp. 648-651.

${ }^{19}$ Scharfe, M. K., Thomas, C. A., Scharfe, D. B., Gascon, N., Cappelli, M. A., and Fernandez, E., "Shear-based model for electron transport in hybrid Hall thruster simulations," IEEE Transactions on Plasma Science, Vol. 36, No. 5 Part 1, 2008, pp. 2058-2068.

${ }^{20}$ Nelson, A. H. and Haines, M. G., "Analysis of the nature and growth of electrothermal waves," Plasma Physics, Vol. 11, No. 10, 1969, pp. 811-837.

${ }^{21}$ Alfvén, H., "Collision between a Nonionized Gas and a Magnetized Plasma," Reviews of Modern Physics, Vol. 32, No. 4, 1960 , pp. 710.

${ }^{22}$ Bohm, D., Burhop, E. H. S., and Massey, H. S. W., "The Use of Probes for Plasma Exploration in Strong Magnetic Fields," The Characteristics of Electrical Discharges in Magnetic Fields, edited by A. Guthrie and R. Wakerling, McGraw-Hill, 1949.

${ }^{23}$ Mikellides, I. G., Katz, I., Hofer, R. R., and Goebel, D. M., "Hall-Effect Thruster Simulations with 2-D Electron Transport and Hydrodynamics Ions," 31 st International Electric Propulsion Conference, IEPC-2009-114, 2009.

${ }^{24}$ Yoshikawa, S. and Rose, D. J., "Anomalous Diffusion of a Plasma across a Magnetic Field," Physics of Fluids, Vol. 5, 1962, pp. 334.

${ }^{25}$ Kaufman, H. R., July 2011, Private Communication.

${ }^{26}$ Esipchuk, Y. V. and Tilinin, G. N., "Drift instability in a Hall-current plasma accelerator," Sov. Phys. - Tech. Phys., Vol. 18, 1974, pp. 928.

${ }^{27}$ Chesta, E., Lam, C. M., Meezan, N. B., Schmidt, D. P., and Cappelli, M. A., "A characterization of plasma fluctuations within a Hall discharge," IEEE Transactions on Plasma Science, Vol. 29, No. 4, 2001, pp. 582-591.

${ }^{28}$ Chesta, E., Meezan, N. B., and Cappelli, M. A., "Stability of a magnetized Hall plasma discharge," Journal of Applied Physics, Vol. 89, No. 6, March 2001, pp. 3099-3107.

${ }^{29}$ Meezan, N. B., Hargus, W. A., and Cappelli, M. A., “Anomalous electron mobility in a coaxial Hall discharge plasma," Physical Review E, Vol. 63, No. 2, Jan. 2001, pp. 026410.

${ }^{30}$ Choueiri, E. Y., "Plasma oscillations in Hall thrusters," Physics of Plasmas, Vol. 8, No. 4, 2001, pp. 1411.

${ }^{31}$ Haas, J. and Gallimore, A., "Considerations on the role of the Hall current in a laboratory-model thruster," IEEE Transactions on Plasma Science, Vol. 30, No. 2, 2002, pp. 687-697.

${ }^{32}$ Reid, B. M. and Gallimore, A. D., "Plasma Potential Measurements in the Discharge Channel of a 6-kW Hall Thruster," 44th AIAA/ASME/SAE/ASEE Joint Propulsion Conference E Exhibit, AIAA 2008-5185, 2008.

${ }^{33}$ Reid, B. M. and Gallimore, A. D., "Langmuir Probe Measurements in the Discharge Channel of a 6-kW Hall Thruster," 44th AIAA/ASME/SAE/ASEE Joint Propulsion Conference E Exhibit, AIAA 2008-4920, 2008.

${ }^{34}$ Reid, B., The Influence of Neutral Flow Rate in the Operation of Hall Thrusters, Ph.D. thesis, University of Michigan, Ann Arbor, MI, 2009.

${ }^{35}$ Mikellides, I. G., Katz, I., and Hofer, R. R., "Design of a Laboratory Hall Thruster with Magnetically Shielded Channel Walls, Phase I: Numerical Simulations," 47th AIAA/ASME/SAE/ASEE Joint Propulsion Conference E Exhibit, AIAA 2011-5809, 2011.

${ }^{36}$ Kasa, I., "A circle fitting procedure and its error analysis," IEEE Transactions on Instrumentation and Measurement, Vol. 25, No. 1, 1976, pp. 8-14.

${ }^{37}$ Chernov, N., Circular and Linear Regression: Fitting Circles and Lines by Least Squares, CRC Press, Taylor \& Francis Group, Boca Raton, FL, 2010.

${ }^{38}$ Smith, A. W. and Cappelli, M. A., "Time and space-correlated plasma potential measurements in the near field of a coaxial Hall plasma discharge," Physics of Plasmas, Vol. 16, No. 7, 2009, pp. 073504-11.

${ }^{39}$ Lobbia, R. B. and Gallimore, A. D., "Two-dimensional Time-resolved Breathing Mode Plasma Fluctuation with Hall Thruster Discharge Settings,” 31st International Electric Propulsion Conference, IEPC 2009-106, 2009. 